\title{
Flexural-gravity waves in ice channel with a lead
}

\author{
L. D. Zeng ${ }^{1}$ A. A. Korobkin ${ }^{2} \dagger$ B. Y. $\mathrm{Ni}^{1}$ and Y. Z. Xue ${ }^{1}$ \\ ${ }^{1}$ College of Shipbuilding Engineering, Harbin Engineering University, Harbin 150001, China \\ ${ }^{2}$ School of Mathematics, University of East Anglia, Norwich NR4 7TJ, United Kingdom
}

(Received $\mathrm{xx}$; revised $\mathrm{xx}$; accepted $\mathrm{xx}$ )

The flexural-gravity symmetric waves propagating in an ice channel with a lead of open water in the ice cover are investigated within the linear theory of hydroelasticity. The ice sheets are modelled as either elastic or as rigid plates. Deflections of the elastic ice sheets are described by the linear elastic plate equation. The flexural-gravity waves propagating along the channel and their dispersion relations are obtained by the normal mode method. It is shown that the dispersion relations for elastic ice approach the dispersion relations for rigid ice as the rigidity of ice increases or wave length decreases. The region in the plane ice thickness/wavenumber, where the ice cover can be treated as rigid with a prescribed accuracy, is determined. The effects of the ice thickness and width of the lead on the phase speeds are studied to determine critical speeds of a vehicle moving along the channel. It is shown that wave modes have several critical speeds, which may merge for some parameters of the channel. The presence of the lead significantly changes characteristics of waves in ice channel. Short waves, which propagate in a channel fully covered with ice, weakly depend on gravity and presence of water in the channel. However, short waves propagating in the same channel but with a lead of open water are gravity-dominated waves localised in the lead. We conclude that flexural-gravity waves in an ice channel with a lead are less pronounced than in the channel completely covered by ice.

\section{Introduction}

Ice tanks and laboratory experiments in them are used to design ships and offshore structures for polar regions and to study their performance in icy waters. In experiments with a ship model, a model is towed in a lead of open water made in the ice cover of the tank. The ship model experiments in ice tank are more complicated and expensive than experiments in a water tank. Such experiments should be carefully designed and prepared. Characteristics of the ice tank with a lead should be examined before the experiments, in particular, in term of flexural-gravity waves propagating along the tank. Dispersion relations of these waves, their phase and group speeds should be investigated with particular focus on the so-called critical speeds of the waves, where the phase and group speeds are equal to each other. If a ship moves in a lead at a critical speed, then the ice deflections and the ship resistance are extreme.

In the polar regions, such leads can be natural caused by various natural forces including wind, wave and current, etc. Squire (2007) or can be made by an icebreaker. Leads are important waterways in the polar regions. The leads may be found or can be made in some northern rivers in springtime. Leads on the rivers can prevent floodings.

The problem of linear waves in the presence of an ice lead has received a considerable attention over the last decades (Squire 2007, 2011). Such waves can arrive at an ice

$\dagger$ Email address for correspondence: a.korobkin@uea.ac.uk 
lead from any direction. For waves propagating perpendicular to the ice lead, Chung \& Linton (2005) studied the flexural-gravity waves in two-dimensional formulations using the residue calculus technique. It was found that there were an infinite number of frequencies at which the waves transmit without any loss from one side of the open water lead to the other. Shi et al. (2019) investigated two-dimensional waves scattering by multiple polynyas using a wide spacing approximation method. For waves propagating into the ice lead obliquely, Williams \& Squire (2006) considered a more general case of flexural-gravity waves propagating into three floating Euler-Bernoulli thin elastic plates with different thicknesses. There were no gaps between the plates and the edges of the plates were free of bending stresses and shear forces. The thickness of the central plate can be reduced to zero, so waves propagating through an ice lead can be dealt with directly. Porter (2018) focused on the same problem but for infinite water depth. He confirmed existence of edge waves, which are localized near the ice edges and do not penetrate the ice sheet deeply. For waves propagating along an ice lead, Marchenko (1997) obtained the dispersion relations within the shallow water approximation, where the ice cover was modelled as either rigid plates or as thin semi-infinite elastic plates.

The problems of wave/ice sheet interaction in confined regions, such as rivers and channels, also received attention. In hydraulic engineering, the problems of waves propagating in ice-covered channels are usually considered as one-dimensional without account for conditions of the ice cover connection with the channel walls. Daly (1993) studied an unsteady flow in a rectangular ice-covered channel by using three linearised governing equations. These equations described the mass and momentum conservation and the motion of the ice cover. Daly (1993) found that for waves longer than 20 characteristic lengths of the ice, the wave propagation is not affected by the presence of ice cover. Daly (1995) further investigated the bending stresses in the ice cover and found that the bending stresses are maximum for flexural-gravity waves, length of which is close to $2 \pi l$, where $l$ is the characteristic length of the ice cover. Xia \& Shen (2002) derived a one-dimensional weakly nonlinear fifth-order $\mathrm{KdV}$ equation for shallow water wave propagation in an uniform channel with a floating ice cover. They proved that the cnoidal waves could generate sufficient stresses to fracture the ice cover.

Beltaos (2004) studied one-dimensional long waves of small amplitudes propagating along an ice channel with transverse cracks and intervals of open water. He concluded that "a truncated low-amplitude wave, which is a wave propagating past an edge (or crack) into an undisturbed region, produces generally higher bending stresses than does an infinitely long wave propagating under an edgeless cover. However, the maximum possible stress is the same for both cases, and occurs at the same dimensionless wave number of 1 , or where the wavelength is equal to about six times the characteristic length of the ice cover." A numerical model was derived by Nzokou et al. (2011) to simulate the interaction between dam break waves and ice-covered channel using the one-dimensional Saint-Venant equations representing mass and momentum conservation for water flowing in an ice-covered channel and the one-dimensional "elastic foundation beam equation of a floating ice sheet including the terms representing vertical acceleration and longitudinal shear stress applied to the bottom of ice sheet by the water flowing under it." They found that, for the conditions of their calculations, ice stiffness had no significant effect on the celerity of waves and their attenuation for waves longer than $400 \mathrm{~m}$. For short waves $(50 \mathrm{~m})$, the decrease in celerity due to stiffness was found to be $6 \%$ and the additional attenuation as high as 20\%. Fuamba et al. (2007) studied dam break wave propagation in a channel partially covered with ice between two consecutive dams. One-dimensional flow in open water part of the channel and under the rigid ice cover were calculated numerically without account for the ice deflection. Then the one-dimensional pressure 
wave under the rigid cover was applied to a finite element model of flexible ice clamped to the channel side walls. The numerical results were in good agreement with the results of laboratory tests, which confirmed the approximation of the rigid ice and the pressure wave propagating under the ice cover. The bending stresses were found to be maximum at the walls of the channel. It was argued that the connection of the ice with the walls was important for the stress distribution in the ice cover.

Nzokou et al. (2009) numerically studied a given incoming one-dimensional water wave propagating in an ice-covered channel, where the ice cover was modelled as a twodimensional ice sheet. The ice sheet was assumed to be clamped to or detached from the channel walls. It was found that there was a high stress concentration near the walls due to the fixed boundary conditions. However, close to the central axis of the channel, the calculated stresses turned out to be similar in value with those obtained using the one-dimensional approximation, that is, without connections of ice cover with the channel walls being considered. Guyenne \& Parau (2017) performed two-dimensional numerical simulations of long nonlinear waves propagating in fragmented sea ice featuring floes and leads. It was observed that gravity-dominated short waves are widespread in areas of open water between floes.

Three-dimensional flexural-gravity waves propagating in the ice-covered channel attracted researchers' attention only recently. Korobkin et al. (2014) investigated hydroelastic waves propagating along a rectangular frozen channel and their dispersion relations for the continuous ice sheet clamped to the walls of the channel. The linear theory of hydroelasticity and the normal mode method were used. The wave profiles across the channel and the strain distributions in the ice sheet were analyzed. It was found that the maximum strains were achieved at the walls for longer waves, and at the centerline of the channel for shorter waves. Batyaev \& Khabakhpasheva (2015) studied similar problem but with free edge conditions between the ice cover and the channel walls. Ren et al. (2020) developed an efficient procedure to the problem of waves propagating in a rectangular channel fully covered by the ice sheet with or without a longitudinal crack in the ice cover. Various edge conditions between the ice sheet and the channel walls were considered. It was shown that the first natural frequency of waves propagating in a channel covered by continuous ice sheet, corresponding to different edge conditions between the ice cover and the channel walls, were very close to each other. For waves propagating in fully ice-covered channel with an infinite longitudinal crack in the ice cover, the effects of the position of the crack on dispersion relations, wave profiles and strain distributions were investigated. It was found that when the crack approaches a channel wall, the results tend to those for the continuous ice sheet with a free edge between the channel wall and ice sheet.

In the present work, we focus on waves propagating along an ice channel with a lead of open water in the ice cover. The presence of a lead significantly changes the characteristics of the propagating waves. The lead is symmetric about the center line of the ice channel. The identical ice sheets on both sides of the lead are clamped to the walls of the channel. The ice sheets are modelled as either thin elastic plates of constant thickness or as rigid plates. The deflection of the ice sheet is described by the elastic plate equation.

The paper is organized as follows. The formulation of the problem is given in $\S 2$. The normal mode method is applied to solve the problem in $\S 3$. The coupled problem of hydroelastic waves is reduced to a linear system of algebraic equations. The same problem but within the rigid-ice model is studied in $\S 4$. Numerical algorithms and their validations are presented in $\S 5$. Numerical results are provided in $\S 6$ for the dispersion relations of the hydroelastic waves, their phase speeds and critical speeds, as well as the 


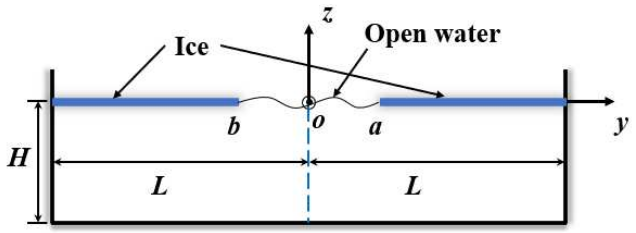

(a)

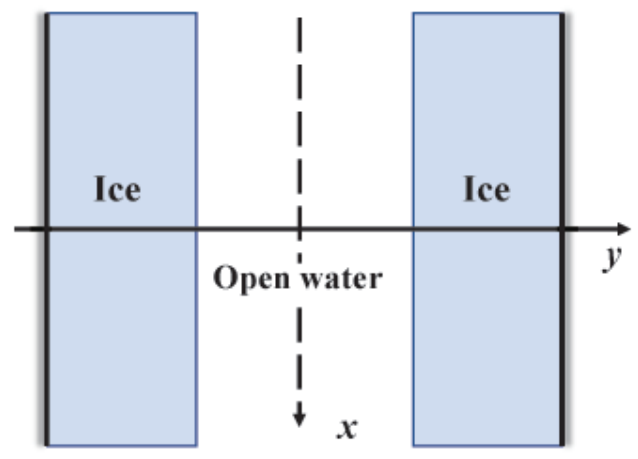

(b)

Figure 1: $(a)$ The front view of the channel with the ice sheets and the open water lead, $(b)$ the top view of the channel.

profiles of these waves across the channel. Conclusions are drawn and future work is discussed in $\S 7$.

\section{Formulation of the problem}

Linear sinusoidal waves propagating along an ice channel with an open water lead in the ice cover are studied. The configuration of the ice tank is shown in figure 1 . The channel, in the Cartesian coordinate system $O x y z$, is of rectangular cross section with width $2 L,-L<y<L$, depth $H,-H<z<0$, and infinite extent in the $x$-direction. The ice cover floating on the water surface is with an open water lead which corresponds to the interval $b<y<a$. The ice is modelled as a thin elastic plate of constant thickness $h$. The mass of the ice per unit area is $m$ and the ice rigidity is $D=E h^{3} /\left[12\left(1-\nu^{2}\right)\right]$ with $E$ and $\nu$ being the Young's modulus and Poisson's ratio of the ice sheet. Within the linear theory of hydroelasticity, the elevation of the upper boundary of the flow region, $z=w(x, y, t)$, is governed by the equations,

$$
\left.\begin{array}{cl}
m w_{t t}+D \nabla^{4} w=p(x, y, 0, t) & (-L<y<b, a<y<L,-\infty<x<\infty), \\
p(x, y, 0, t)=0 \quad(b<y<a,-\infty<x<\infty),
\end{array}\right\}
$$

where $p(x, y, 0, t)=-\rho \varphi_{t}(x, y, 0, t)-\rho g w(x, y, t)$ is the hydrodynamic pressure on the upper boundary of the flow region, $\rho$ is the water density, $g$ is the gravitational acceleration, and $\varphi(x, y, z, t)$ is the velocity potential of the flow. Note that $w(x, y, t)$ is not continuous at $y=b$ and $y=a$. The elevation of the free surface and the deflection of the ice cover are, in general, different at the ice edges. The ice edges are free of stresses and shear forces,

$$
\frac{\partial^{2} w}{\partial y^{2}}+\nu \frac{\partial^{2} w}{\partial x^{2}}=0, \quad \frac{\partial^{3} w}{\partial y^{3}}+(2-\nu) \frac{\partial^{3} w}{\partial x^{2} \partial y}=0 \quad\left(y=b^{-}, a^{+},-\infty<x<\infty\right)
$$

where $y=b^{-}$and $y=a^{+}$imply that we approach the edges from the ice. The other edges of the ice plates, $y= \pm L$, are clamped (frozen) to the vertical walls of the channel, 


$$
w=0, \quad \frac{\partial w}{\partial y}=0 \quad(y= \pm L) .
$$

The velocity potential $\varphi(x, y, z, t)$ satisfies the Laplace equation in the flow region,

$$
\nabla^{2} \varphi=0 \quad(|x|<\infty,|y|<L, \quad-H<z<0),
$$

and the boundary conditions,

$$
\frac{\partial \varphi}{\partial y}=0 \quad(y= \pm L), \quad \frac{\partial \varphi}{\partial z}=0 \quad(z=-H), \quad \frac{\partial \varphi}{\partial z}=\frac{\partial w}{\partial t} \quad(z=0)
$$

where the third equation is linearised. The waves propagating along the ice channel correspond to the solutions of the problem (2.1)-(2.5) in the form

$$
w=F(y) \cos (k x-\omega t), \quad \varphi=\omega \Phi(y, z) \sin (k x-\omega t),
$$

where $k$ is the wavenumber and $\omega$ is the wave frequency, the function $F(y)$ describes the wave profile across the channel. Along the channel the wave is sinusoidal. We shall determine the dispersion relations between the wavenumber $k$ and the wave frequencies $\omega_{n}(k)$, and the corresponding wave profiles $F_{n}(y)$, where $\omega_{n+1}(k)>\omega_{n}(k)$ and $n \geqslant 1$.

Substituting (2.6) in (2.1)-(2.5), we arrive at the boundary-value problem with respect to the function $F(y)$,

$$
\begin{gathered}
D\left[F^{i v}-2 k^{2} F^{\prime \prime}+k^{4} F\right]-m \omega^{2} F+\rho g F=\rho \omega^{2} \Phi(y, 0) \quad(-L<y<b, a<y<L), \\
\rho g F=\rho \omega^{2} \Phi(y, 0) \quad(b<y<a) . \\
F=F^{\prime}=0 \quad(y= \pm L), \quad F^{\prime \prime}-\nu k^{2} F=0 \quad F^{\prime \prime \prime}-(2-\nu) k^{2} F^{\prime}=0 \quad(y=b, y=a) .
\end{gathered}
$$

The first equation in (2.7) is the fourth-order ordinary differential equation for $F(y)$ in the intervals $-L<y<b$ and $a<y<L$ with four boundary conditions (2.8) in each interval. The potential $\Phi(y, z)$ in the right-hand side of $(2.7)$ is the solution of the boundary problem, which follows from (2.4) and (2.5),

$$
\begin{gathered}
\Phi_{y y}+\Phi_{z z}-k^{2} \Phi=0 \quad(|y|<L, \quad-H<z<0), \\
\Phi_{y}=0 \quad(y= \pm L), \quad \Phi_{z}=0 \quad(z=-H), \quad \Phi_{z}=F(y) \quad(z=0) .
\end{gathered}
$$

On the upper boundary of the channel, the pressure caused by the wave (2.6) is given by

$$
\left.\begin{array}{c}
p(x, y, 0, t)=-\rho \varphi_{t}-\rho g w(x, y, t)=P(y) \cos (k x-\omega t), \\
P(y)=\rho \omega^{2} \Phi(y, 0)-\rho g F(y) .
\end{array}\right\}
$$

We shall determine non-trivial solutions of the homogeneous boundary problems (2.7)(2.10) and the corresponding frequencies $\omega_{n}(k)$ within the model of elastic ice $(2.7),(2.8)$ and the model of rigid ice, where $F(y)=0$ in $-L<y<b$ and $a<y<L$. 


\section{Waves within elastic ice model}

The ice deflection is presented by superposition,

$$
F(y)= \begin{cases}\sum_{n=1}^{\infty} G_{n} \chi_{n}(y) & (-L<y<b) \\ \sum_{n=1}^{\infty} F_{n} \psi_{n}(y) & (a<y<L)\end{cases}
$$

Substituting equation (3.1) in the first equation of (2.7) without the inhomogeneous term and using the boundary conditions (2.8), we arrive the eigen-value problem of the modes $\chi_{n}(y)$ and $\psi_{n}(y)$,

$$
\left\{\begin{array} { c } 
{ ( \frac { \mathrm { d } ^ { 2 } } { \mathrm { d } y ^ { 2 } } - k ^ { 2 } ) ^ { 2 } \chi _ { n } = \beta _ { n } ^ { 4 } \chi _ { n } \quad ( - L < y < b ) , } \\
{ \chi _ { n } ^ { \prime \prime } ( b ) = \nu k ^ { 2 } \chi _ { n } ( b ) , \quad \chi _ { n } ( - L ) = 0 , } \\
{ \chi _ { n } ^ { \prime \prime \prime } ( b ) = ( 2 - \nu ) k ^ { 2 } \chi _ { n } ^ { \prime } ( b ) , \quad \chi _ { n } ^ { \prime } ( - L ) = 0 , }
\end{array} \left\{\begin{array}{c}
\left(\frac{\mathrm{d}^{2}}{\mathrm{~d} y^{2}}-k^{2}\right)^{2} \psi_{n}=\lambda_{n}^{4} \psi_{n} \quad(a<y<L), \\
\psi_{n}^{\prime \prime}(a)=\nu k^{2} \psi_{n}(a), \quad \psi_{n}(L)=0, \\
\psi_{n}^{\prime \prime \prime}(a)=(2-\nu) k^{2} \psi_{n}^{\prime}(a), \quad \psi_{n}^{\prime}(L)=0,
\end{array}\right.\right.
$$

with the corresponding eigen values $\beta_{n}$ and $\lambda_{n}$, which are obtained in Appendix A. The modes $\chi_{n}(y)$ and $\psi_{n}(y)$ are orthogonal and normalised,

$$
\int_{-L}^{b} \chi_{n}(y) \chi_{m}(y) \mathrm{d} y=\delta_{n m}, \quad \int_{a}^{L} \psi_{n}(y) \psi_{m}(y) \mathrm{d} y=\delta_{n m},
$$

where $\delta_{n n}=1$ and $\delta_{n m}=0$ for $n \neq m$. The coefficients $G_{n}$ and $F_{n}$ in (3.1) are unknown in advance and should be determined using the equations (2.7)-(2.10).

Equations $(2.7),(2.11)$ and $(3.2)$ provide the pressure $P(y)$ on the ice-water interface,

$$
P(y)=\left\{\begin{array}{cc}
\sum_{n=1}^{\infty} G_{n} \chi_{n}(y)\left[-m \omega^{2}+D \beta_{n}^{4}\right] & (-L<y<b), \\
\sum_{n=1}^{\infty} F_{n} \psi_{n}(y)\left[-m \omega^{2}+D \lambda_{n}^{4}\right] & (a<y<L) .
\end{array}\right.
$$

In the lead, $b<y<a$, the pressure is zero, $P(y)=0$. The kinematic condition, $\Phi_{z}(y, 0)=$ $F(y)$, makes it possible to transform equations (2.7) to the following boundary condition on the upper boundary $z=0$,

$$
\frac{\partial \Phi}{\partial z}-\frac{\omega^{2}}{g} \Phi=\left\{\begin{array}{cc}
\sum_{n=1}^{\infty} G_{n} \chi_{n}(y)\left[\frac{m \omega^{2}}{\rho g}-\frac{D \beta_{n}^{4}}{\rho g}\right] & (-L<y<b), \\
0 & (b<y<a), \\
\sum_{n=1}^{\infty} F_{n} \psi_{n}(y)\left[\frac{m \omega^{2}}{\rho g}-\frac{D \lambda_{n}^{4}}{\rho g}\right] & (a<y<L)
\end{array}\right.
$$

The solution of the problem (2.9)-(2.10), where the condition at $z=0$ is changed to (3.5), can be written as

$$
\Phi(y, z)=\sum_{n=1}^{\infty} \Phi_{n}(y, z)
$$


where the potentials $\Phi_{n}(y, z), n \geqslant 1$, are the solutions of the following boundary value problem

$$
\begin{gathered}
\Phi_{n, y y}+\Phi_{n, z z}-k^{2} \Phi_{n}=0 \quad(|y|<L, \quad-H<z<0), \\
\Phi_{n, y}=0 \quad(y= \pm L), \quad \Phi_{n, z}=0 \quad(z=-H), \\
\frac{\partial \Phi_{n}}{\partial z}-\frac{\omega^{2}}{g} \Phi_{n}=\left\{\begin{array}{cc}
G_{n} \chi(y) \frac{m \omega^{2}-D \beta_{n}^{4}}{\rho g} & (z=0,-L<y<b), \\
0 \quad & (z=0, b<y<a), \\
F_{n} \psi_{n}(y) \frac{m \omega^{2}-D \lambda_{n}^{4}}{\rho g} & (z=0, a<y<L) .
\end{array}\right.
\end{gathered}
$$

The kinematic condition, $\Phi_{z}(y, 0)=F(y)$, where $-L<y<L$, equations (3.1) and (3.6) provide the equation,

$$
\sum_{n=1}^{\infty} \frac{\partial \Phi_{n}}{\partial z}= \begin{cases}\sum_{n=1}^{\infty} G_{n} \chi(y) & (-L<y<b), \\ \sum_{n=1}^{\infty} F_{n} \psi_{n}(y) & (a<y<L) .\end{cases}
$$

which leads to the infinite linear system of equations for the coeffcients $G_{n}$ and $F_{n}$,

$$
\left\{\begin{array}{cl}
\sum_{n=1}^{\infty} \int_{-L}^{b} \frac{\partial \Phi_{n}}{\partial z} \chi_{k}(y) \mathrm{d} y=G_{n} \delta_{k n} & (-L<y<b), \\
\sum_{n=1}^{\infty} \int_{a}^{L} \frac{\partial \Phi_{n}}{\partial z} \psi_{k}(y) \mathrm{d} y=F_{n} \delta_{k n} \quad(a<y<L),
\end{array}\right.
$$

using the orthogonality relation (3.3). Fianlly we solve the homogeneous equation (3.11) to determine the coeffcients $G_{n}$ and $F_{n}$.

In the present study, we limit ourselves to the open water leads which are symmetric with respect to the central line of the channel, $-b=a$, and symmetric waves, $w(x,-y, t)=w(x, y, t)$. Then, we have $G_{n}=F_{n}, \beta_{n}=\lambda_{n}$ and $\chi(-y)=\psi(y)$. Therefore, the solution of the problem (2.9)-(2.10) can be thought as

$$
\Phi(y, z)=\sum_{n=1}^{\infty} F_{n}\left[\frac{m \omega^{2}}{\rho g}-\frac{D \lambda_{n}^{4}}{\rho g}\right] \Phi_{n}(y, z),
$$

where the condition at $z=0$ for the potential $\Phi_{n}(y, z)$ can be rewritten as

$$
\frac{\partial \Phi_{n}}{\partial z}-\frac{\omega^{2}}{g} \Phi_{n}=\left\{\begin{array}{cc}
\psi_{n}(|y|) & (z=0, a<|y|<L) \\
0 & (z=0,-a<y<a) .
\end{array}\right.
$$

The kinematic condition, $\Phi_{z}(y, 0)=F(y)$, where $-L<y<L$, the second equation of (3.1) and equation (3.12) provide the equation,

$$
\sum_{n=1}^{\infty} F_{n}\left[\frac{m \omega^{2}}{\rho g}-\frac{D \lambda_{n}^{4}}{\rho g}\right] \frac{\partial \Phi_{n}}{\partial z}(y, 0)=\sum_{n=1}^{\infty} F_{n} \psi_{n}(y) \quad(a<y<L) .
$$


Multiplying both sides of (3.14) by $\psi_{k}(y)$ and integrating the results in $y$ from $a$ to $L$ using the second equation of (3.3), we find

$$
\sum_{n=1}^{\infty} F_{n} \frac{m \omega^{2}-D \lambda_{n}^{4}}{\rho g} \int_{a}^{L} \frac{\partial \Phi_{n}}{\partial z}(y, 0) \psi_{k}(y) \mathrm{d} y=F_{n} \delta_{k n}
$$

Equations (3.13) and the second equation of (3.3) provide

where

$$
\int_{a}^{L} \frac{\partial \Phi_{n}}{\partial z}(y, 0) \psi_{k}(y) \mathrm{d} y=\delta_{k n}+\frac{\omega^{2}}{g} \tilde{A}_{k n}
$$

$$
\tilde{A}_{k n}=\tilde{A}_{n k}=\int_{a}^{L} \Phi_{n}(y, 0) \psi_{k}(y) \mathrm{d} y .
$$

It is convenient to denote $\left|m \omega^{2}-D \lambda_{n}^{4}\right| /(\rho g)$ by $B_{n}$ and introduce new unknown coefficients $\tilde{F}_{n}=e_{n} \sqrt{B_{n}} F_{n}$, where $e_{n}=1$ for $\left(m \omega^{2}-D \lambda_{n}^{4}\right)>0$, and $e_{n}=-1$ for $\left(m \omega^{2}-D \lambda_{n}^{4}\right)<0$. Then equation (3.15) provides the following symmetric system

$$
\sum_{n=1}^{\infty} C_{k n} \tilde{F}_{n}=e_{k} \tilde{F}_{k}
$$

where

$$
C_{k n}=C_{n k}=B_{k} \delta_{k n}+\frac{\omega^{2}}{g} \sqrt{B_{n} B_{k}} \tilde{A}_{k n}
$$

To avoid the problem with large coefficients of the system (3.16), we define $\tilde{B}_{n}=$ $\rho g B_{n} /\left(D \lambda_{n}^{4}\right)$, which are finite for large $n$, and new unknowns $\hat{F}_{n}=D \lambda_{n}^{2} \tilde{F}_{n} /(\rho g)$. The equation (3.16) yields,

$$
\boldsymbol{A}^{*} \hat{\boldsymbol{F}}=0
$$

where $A_{k n}^{*}=\frac{\omega^{2}}{g} \sqrt{\tilde{B}_{n} \tilde{B}_{k}} \tilde{A}_{k n}+\left(\tilde{B}_{n}-\frac{\rho g}{D \lambda_{n}^{4}} e_{n}\right) \delta_{k n}$ and $\hat{\boldsymbol{F}}=\left(\hat{F}_{1}, \hat{F}_{2}, \hat{F}_{3}, \ldots \ldots\right)^{T}$. The matrix $\boldsymbol{A}^{*}$ is symmetric.

The determinant of the system (3.17) depends on $\omega$ and $k$. For each wave number $k$ we can calculate the determinant as a function of $\omega$ and find roots of this function. The roots provide the dispersion relations $\omega=\omega_{n}(k)$, where $\omega_{n+1}(k)>\omega_{n}(k)$ and $n \geqslant 1$. Finally we solve the homogeneous system (3.17) to determine $F_{n}$ and finally $F_{n}$. The integrals $\tilde{A}_{k n}(k, \omega)$ are evaluated in Appendix B.

Note that the roots $\omega_{n}(k)$, which are the frequencies of waves propagating in the channel partly covered with ice, cannot be equal to the frequencies $\omega_{m}^{(f)}(k)=$ $\sqrt{g \sqrt{k^{2}+\mu_{m}^{2}} \tanh \left[\sqrt{k^{2}+\mu_{m}^{2}} H\right]}$, where $\mu_{m}=\pi(m-1) / L$ and $m \geqslant 1$, of waves in the same channel but without ice in it. Therefore, we do not need to calculate the determinant of the system (3.17) for $\omega$ in vicinities of $\omega_{m}^{(f)}(k)$, see Appendix B.

The deflection of ice is given by (3.1). The wave profile in the lead follows from kinematic condition, $F(y)=\Phi_{z}(y, 0)$, where $\Phi_{z}(y, 0)$ is provided by (3.12) and (3.13). The velocity potentials $\Phi_{n}(y, z)$ are evaluated in Appendix B. Finally the deflection of the whole upper surface, $F(y)$, where $-L<y<L$, is normalised by the condition that the maximum deflection is equal to 1 .

\section{Symmetric waves within rigid ice model}

The problem (2.6)-(2.11) in the symmetric case for the rigid ice model reads 


$$
\begin{gathered}
\Phi_{y y}+\Phi_{z z}-k^{2} \Phi=0 \quad(|y|<L, \quad-H<z<0), \\
\Phi_{y}=0 \quad(y= \pm L, \quad-H<z<0), \\
\Phi_{z}=0 \quad(z=-H, \quad|y|<L), \\
\Phi_{z}=0 \quad(z=0, \quad a<|y|<L), \\
\Phi_{z}=F(y), \quad F(y)=\frac{\omega^{2}}{g} \Phi(y, 0) \quad(z=0, \quad|y|<a),
\end{gathered}
$$

where $F(y)$ describes now the wave profile across the open water part of the ice channel. The conditions (4.5) are the kinematic and dynamic, $P(y)=0$, conditions in the region of open water. We shall determine the wave profiles $F_{n}(y)$ and the corresponding frequencies $\omega_{n}(k)$, where $\omega_{n+1}(k) \geqslant \omega_{n}(k)$, for $k \geqslant 0$. In this section, we consider only symmetric profiles with $F(-y)=F(y)$.

The method of separating variables applied to (4.1)-(4.4) provides

$$
\Phi(y, z)=\sum_{n=0}^{\infty} C_{n} \cosh \left[æ_{n}(z+H)\right] \cos \left(\lambda_{n} y\right),
$$

where $\lambda_{n}=n \pi / L$ and $æ_{n}=\sqrt{\lambda_{n}^{2}+k^{2}}$ for $n \geqslant 0$. The coefficients are related to the function $F(y)$ by the kinematic condition at $z=0$,

$$
\Phi_{z}(y, 0)=0 \quad(a<|y|<L), \quad \Phi_{z}(y, 0)=F(y) \quad(|y|<a) .
$$

The functions $\left\{\cos \left(\lambda_{n} y\right)\right\}_{n=0}^{\infty}$ are orthogonal in the interval $-L<y<L$,

$$
\int_{-L}^{L} \cos \left(\lambda_{n} y\right) \cos \left(\lambda_{m} y\right) \mathrm{d} y=L \varepsilon_{m} \delta_{n m},
$$

where $\varepsilon_{0}=2$ and $\varepsilon_{m}=1$ for $m \geqslant 1, \delta_{n m}=1$ for $n=m, \delta_{n m}=0$ for $n \neq m$.

Multiplying both sides of (4.7) by $\cos \left(\lambda_{m} y\right)$ and integrating in $y$ from $-L$ to $L$ using (4.6) and (4.8), we find

$$
C_{m} æ_{m} \sinh \left(æ_{m} H\right) \varepsilon_{m} L=\int_{-a}^{a} F(y) \cos \left(\lambda_{m} y\right) \mathrm{d} y .
$$

Any even function in the symmetric interval $(-a, a)$ can be presented by its Fourier series,

$$
F(y)=\sum_{k=0}^{\infty} F_{k} \cos \left(\frac{k \pi}{a} y\right) .
$$

Substituting (4.10) in (4.9) and denoting the integrals $\frac{1}{L} \int_{-a}^{a} \cos \left(\frac{k \pi}{a} y\right) \cos \left(\frac{m \pi}{L} y\right) \mathrm{d} y$ by $S_{k m}$, we find

$$
C_{m}=\frac{1}{æ_{m} \varepsilon_{m} \sinh \left(æ_{m} H\right)} \sum_{k=0}^{\infty} F_{k} S_{k m} .
$$

The dynamic condition (4.5) together with (4.6), (4.10) and (4.11) provides the following equality 


$$
\sum_{k=0}^{\infty} F_{k} \cos \left(\frac{k \pi}{a} y\right)=\frac{\omega^{2}}{g} \sum_{m=0}^{\infty} F_{m} \sum_{n=0}^{\infty} \frac{S_{m n} \cos \left(\lambda_{n} y\right)}{æ_{n} \varepsilon_{n} \tanh \left(æ_{n} H\right)},
$$

where $-a<y<a$. Multiplying both parts of (4.12) by $\cos \left(\frac{q \pi}{a} y\right)$ and integrating in $y$ from $-a$ to $a$ using (4.8), where $L$ is changed to $a$, we obtain

$$
\varepsilon_{q} a F_{q}=\frac{\omega^{2} L}{g} \sum_{m=0}^{\infty} F_{m} \sum_{n=0}^{\infty} \frac{S_{m n} S_{q n}}{æ_{n} \varepsilon_{n} \tanh \left(æ_{n} H\right)} .
$$

Introducing a symmetric matrix $\boldsymbol{A}$ with the dimensionless elements

$$
A_{m q}=\sum_{n=0}^{\infty} \frac{S_{m n} S_{q n}}{\varepsilon_{n} \sqrt{\varepsilon_{m} \varepsilon_{q}} æ_{n} a \tanh \left(æ_{n} H\right)},
$$

and the dimensionless spectral parameter $\Omega=\omega^{2} L / g$, the system (4.13) can be written in the matrix form

$$
(\boldsymbol{I}-\Omega \boldsymbol{A}) \boldsymbol{F}=0,
$$

where the coefficients $\boldsymbol{F}=\left\{\sqrt{2} F_{0}, F_{1}, F_{2}, \ldots\right\}$.

The dispersion relation of the waves propagating between two rigid ice plates are obtained as the roots of the equation

$$
\operatorname{det}|\boldsymbol{I}-\Omega \boldsymbol{A}|=0 \text {. }
$$

Then we calculate the corresponding eigen vectors $\boldsymbol{F}$ of system (4.15) and normalise them in such a way that the function $F(y)$ given by (4.10) in the interval $(-a, a)$ has its absolute maximum value, $\max _{-a \leqslant y \leqslant a}|F(y)|$, equal to one.

The elements $A_{m q}$ of the matrix $\boldsymbol{A}$, which are given by (4.14), depend on the aspect ratios $a / L=\sigma, H / L=\delta$ and the dimensionless wave number $k H=æ$. The integrals $S_{k m}$ are evaluated under the assumption that $a / L=\sigma$ is not a rational number. This means there are no such integer $k$ and $m$ that $\sigma=k / m$. We have $S_{00}=2 a / L$, where both $k=0$ and $m=0$, and $S_{k 0}=0$. In other cases,

$$
S_{k m}=\frac{1}{2 L} \int_{-a}^{a}\left\{\cos \left[\left(\frac{k}{a}+\frac{m}{L}\right) \pi y\right]+\cos \left[\left(\frac{k}{a}-\frac{m}{L}\right) \pi y\right]\right\} \mathrm{d} y,
$$

where $(k / a+m / L)>0$, and $(k / a-m / L) \neq 0$. Then

$$
S_{k m}=\frac{2 \sigma}{\pi}(-1)^{k+1} \sin (m \pi \sigma) \frac{m \sigma}{k^{2}-(m \sigma)^{2}} .
$$

Note that there is zero contribution from $n=0$ in (4.14) because of $S_{k 0}=0$, and $\varepsilon_{n}=1$ for $n \geqslant 1$. Substituting (4.17) in (4.14), we find

$$
A_{m q}=\frac{4}{\pi^{2}} \frac{(-1)^{m+q}}{\sqrt{\varepsilon_{m} \varepsilon_{q}}} \frac{\sigma}{\delta} \sum_{n=1}^{\infty} \frac{n^{2} \sin ^{2}(n \pi \sigma)}{\left[n^{2}-m^{2} / \sigma^{2}\right]\left[n^{2}-q^{2} / \sigma^{2}\right]} \frac{1}{\tilde{\mathfrak{x}}_{n} \tanh \tilde{\mathfrak{x}}_{n}},
$$

where $\tilde{æ}_{n}=æ_{n} H=\sqrt{\lambda_{n}^{2} H^{2}+k^{2} H^{2}}=\sqrt{n^{2} \pi^{2} \delta^{2}+\mathfrak{x}^{2}}$. For large $n$,

$$
\frac{1}{\tilde{æ}_{n} \tanh \left(\tilde{æ}_{n}\right)}=\frac{1}{n \pi \delta}+\frac{1}{\pi \delta} \kappa_{n}
$$


where $\kappa_{n}=O\left(n^{-3}\right)$ as $n \rightarrow \infty$. Equations (4.18) and (4.19) provide

$A_{m q}=\frac{2}{\pi^{3}} \frac{(-1)^{m+q}}{\sqrt{\varepsilon_{m} \varepsilon_{q}}} \frac{\sigma}{\delta^{2}}\left\{\sum_{n=1}^{\infty} \frac{n^{2} \kappa_{n}(1-\cos (2 n \pi \sigma))}{\left[n^{2}-m^{2} / \sigma^{2}\right]\left[n^{2}-q^{2} / \sigma^{2}\right]}+\sum_{n=1}^{\infty} \frac{n(1-\cos (2 n \pi \sigma))}{\left[n^{2}-m^{2} / \sigma^{2}\right]\left[n^{2}-q^{2} / \sigma^{2}\right]}\right\}$.

The elements of the first series decay as $n^{-5}$ as $n \rightarrow \infty$. Both the first and second series are calculated numerically.

Equations (4.15) and (4.10) provide the dispersion relations and the profiles of waves propagating in the open water lead. The numerical algorithm is the same as for equation (3.17), which is for elastic ice model. The frequencies of the waves propagating along the channel within the rigid-ice model are denoted by $\omega_{n}^{(r)}(k)$, where $\omega_{n}^{(r)}(k)<\omega_{n+1}^{(r)}(k)$ for $n \geqslant 1$.

\section{Numerical algorithms and their validation}

\subsection{Numerical algorithms}

Within the elastic ice model, see section 3, we should find the non-trival solutions of the infinite algebraic system $(3.17), \hat{\boldsymbol{F}}_{n}(k)=\left(\hat{F}_{n, 1}(k), \hat{F}_{n, 2}(k), \ldots\right)^{T}$, and the corresponding frequencies $\omega_{n}(k)$ for wavenumbers $k$ from an interval $\left(0, k^{*}\right)$. Here $\omega_{n+1}(k)>\omega_{n}(k)$, where $0<k<k^{*}$ and $n \geqslant 1$. We limit ourselves to five lowest modes, $n=1,2,3,4,5$, and long waves with $k^{*}=2 \mathrm{~m}^{-1}$, which includes waves longer than $3 \mathrm{~m}$. The frequencies $\omega_{n}(k)$ are roots of the determinant $\left|\boldsymbol{A}^{*}(k, \omega)\right|$. This determinant is approximated by the determinant $\left|\boldsymbol{A}_{N}^{*}(k, \omega)\right|$ of the truncated matrix $\boldsymbol{A}_{N}^{*}$, where first $N$ rows and columns of matrix $\boldsymbol{A}^{*}(k, \omega)$ are retained. For each $k$ from an interval $\left(0, k^{*}\right)$ with step $\Delta k=0.02 \mathrm{~m}^{-1}$, the determinant $\left|\boldsymbol{A}_{N}^{*}(k, \omega)\right|$ is calculated for frequencies starting from zero with the step $0.02 \mathrm{~s}^{-1}$ until the intervals of the five roots of the determinant are determined. Then the roots $\omega_{n}^{(N)}(k)$ of the equation $\left|\boldsymbol{A}_{N}^{*}(k, \omega)\right|=0$ are calculated by the bisection method, which narrows an interval that contains a root down to $1 \times 10^{-5} \mathrm{~s}^{-1}$. The convergence analysis of the roots $\omega_{n}^{(N)}(k)$ with respect to the truncation size $N$ was investigated for $n=1$ and $n=2$, as shown in figure 2. We can find that the difference of $\omega_{n}^{(N)}(k)$ becomes smaller as $N$ increase for $n=1$ and $n=2$. Particularly, it was obtained that $\left(\omega_{1}^{(500)}(k)-\omega_{1}^{(400)}(k)\right)$ is less than $10^{-3} \mathrm{~s}^{-1}$, where $0<k<0.8 \mathrm{~m}^{-1}$. Below, $\omega_{n}(k)$ are approximated by $\omega_{n}^{(500)}(k)$ for elastic ice, $n=1,2,3,4,5$ and $0<k<k^{*}$. For each $k, n$ and correspondingly $\omega_{n}(k)$, the truncated system (3.17) with the matrix $\boldsymbol{A}_{N}^{*}\left(k, \omega_{n}(k)\right)$ is solved with respect to the vector $\hat{\boldsymbol{F}}_{n}^{(N)}(k)=\left(\hat{F}_{n, 1}^{(N)}(k), \hat{F}_{n, 2}^{(N)}(k), \ldots\right)^{T}$, which provides the wave profile across the channel using (3.1). To calculate the non-trival solutions of the truncated system with zero determinant, we find an $i$-th column of $\boldsymbol{A}_{N}^{*}\left(k, \omega_{n}(k)\right)$ with largest $\sum_{j=1}^{N}\left(\boldsymbol{A}_{N}^{*}\right)_{i j}^{2}$, set $\hat{F}_{i}^{(N)}=1$ and move this column to the right hand side of the system. Next we delete the $i$-th row from the system to keep the resulting nonhomogeneous system symmetric. Note that the obtained vector-solution with $\hat{F}_{i}^{(N)}=1$ being multiplied by any constant is also a solution of the truncated system. A constant is selected for each $k$ and $n$ in such a way that the maximum magnitude of the $n$-th wave elevation across the channel including the lead is equal to 1 . The wave amplitude is not determined in the present problem.

For the model of rigid ice, see section 4, the system (4.16) is solved using the algorithm described above for elastic ice with the only difference: it was obtained that truncation of 


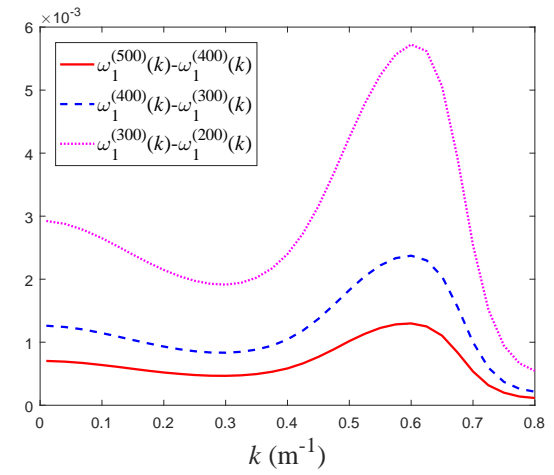

(a)

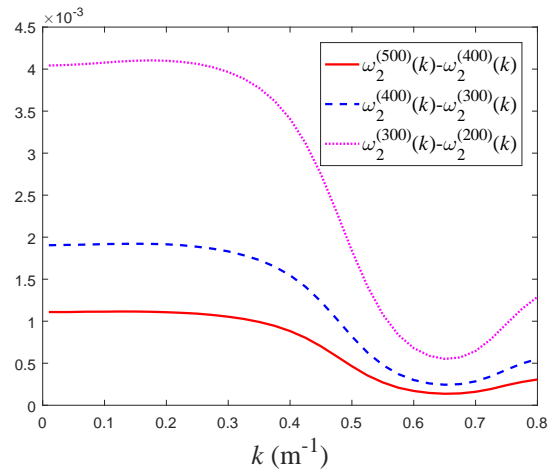

(b)

Figure 2: Convergence analysis of the roots $\omega_{n}^{(N)}(k)$ with respect to $N$ for $a=1.1 \mathrm{~m}, L=12 \mathrm{~m}, H=5 \mathrm{~m}, h=0.05 \mathrm{~m}, n=1$ (a) and $n=2$ (b).

the matrix in (4.16) with $N=150$ provides the same accuracy of the results as $N=500$ in the model of elastic ice.

\subsection{Validation of the algorithm}

For a shrinking lead, $a \rightarrow 0$, the present problem describes waves propagating in the frozen channel with the longitudinal crack at the centre of the ice cover. The latter problem was studied by Ren et al. (2020) for the following conditions: $H=5 \mathrm{~m}, L=10 \mathrm{~m}$, $h=0.1 \mathrm{~m}, \rho_{i}=917 \mathrm{~kg} \mathrm{~m}{ }^{-3}, E=4.2 \times 10^{9} \mathrm{~N} \mathrm{~m}^{-2}, \nu=0.3, \rho=1000 \mathrm{~kg} \mathrm{~m}{ }^{-3}$, $g=9.81 \mathrm{~m} \mathrm{~s}^{-2}$. Dispersion relations for these conditions of symmetric waves with $n=1$ and $n=2$, which are obtained by the present algorithm with $a=0$, are shown in figure 3 together with the dispersion relations for the same waves computed by Ren et al. (2020), where $\omega^{*}=\omega / \sqrt{g / H}$. It is seen that the results are in a very good agreement.

\section{Numerical results}

The results of the present study are presented in terms of dispersion relations, phase and critical speeds, profiles and strains of the symmetric waves propagating along the channel with a lead. Numerical calculations are performed for a freshwater ice with density $\rho_{i}=917 \mathrm{~kg} \mathrm{~m}^{-3}$, Young's modulus $E=4.2 \times 10^{9} \mathrm{~N} \mathrm{~m}^{-2}$ and Poisson's ratio $\nu=0.3$. The thickness $h$ of the ice sheet is varied from $0.5 \mathrm{~mm}$ to $2 \mathrm{~m}$. The half-width $L$ of the channel is $12 \mathrm{~m}$ and the water depth $H$ is $5 \mathrm{~m}$. The half-width $a$ of the lead is varied from $0 \mathrm{~m}$ (a crack) to $6 \mathrm{~m}$. Water density is $\rho=1000 \mathrm{~kg} \mathrm{~m}^{-3}$ and the gravity acceleration is $g=9.8 \mathrm{~m} \mathrm{~s}^{-2}$. Dimensions of the channel, $L$ and $H$, and the ice characteristics, $\rho_{i}$, $E$ and $\nu$ are constant in this analysis. Dimensionless variables and parameters are used below. They are denoted by an asterisk, $k^{*}=k H, \omega^{*}=\omega / \sqrt{g / H}, h^{*}=h / H, a^{*}=a / H$, $L^{*}=L / H, c^{*}=c / \sqrt{g H}, \lambda^{*}=\lambda / H, \Phi^{*}=\Phi / H^{2}$.

\subsection{Dispersion relations}

The dispersion relations, $\omega^{*}=\omega_{n}^{*}\left(k^{*}\right)$, of hydroelastic waves in an ice channel with a lead are expected to converge to the corresponding dispersion relations of waves in the same channel without ice, $\omega_{n}^{*(f)}\left(k^{*}\right)$, which are defined in section 3 , as the ice thickness decreases, and to the dispersion relations of waves in the same channel with rigid ice, 


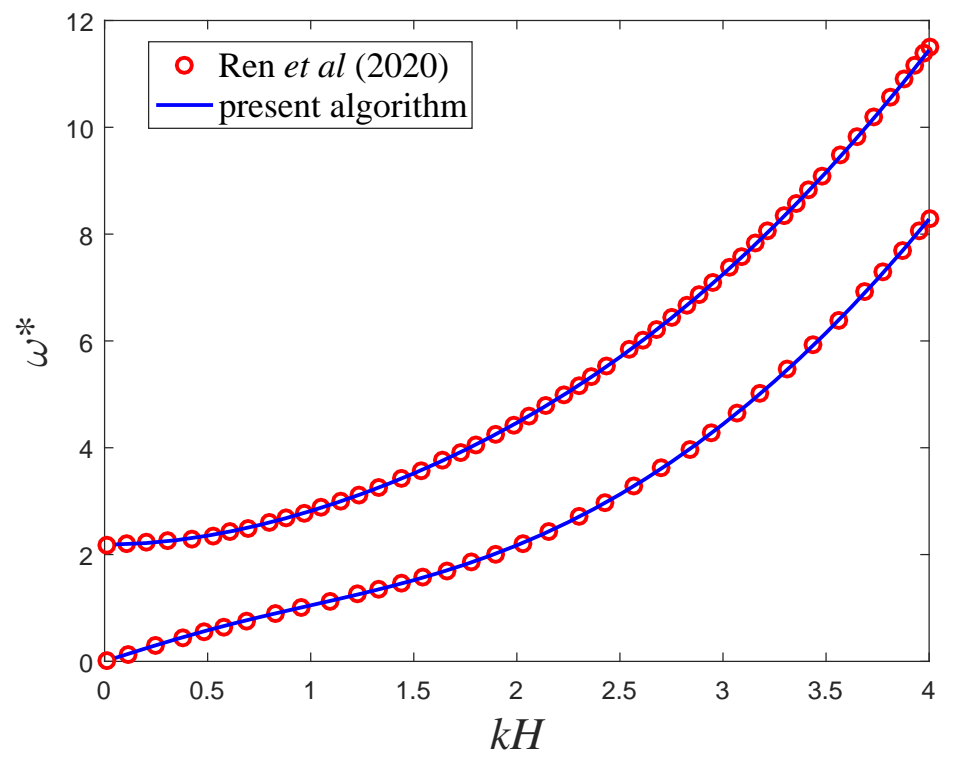

Figure 3: Dispersion relations for first two symmetric hydroelastic waves by Ren et al. (2020) (circle markers) and by the present algorithm (solid lines).

$\omega_{n}^{*(r)}\left(k^{*}\right)$, which are defined in section 4 , as the ice thickness increases. Figure 4 showns dispersion relations of waves with $n=1,2,3,4,5$ for $h^{*}=4 \times 10^{-3}, 1 \times 10^{-3}, 1 \times 10^{-4}$ and for the same channel without ice. The half-width of the lead is $a^{*}=0.22$ in this figure. It is seen that the wave frequencies $\omega_{n}^{*}\left(k^{*}\right)$ increase with increase of the ice thickness for given $n$ and $k^{*}$. Higher wave frequencies are more sensitive to the ice thickness than the low frequencies with $n=1$ and 2 , see figure 4(b). The dispersion relations of hydroelastic waves for $h^{*}=1 \times 10^{-4}$ are close to the corresponding dispersion relations without ice, see figure $4(\mathrm{a}),(\mathrm{b})$. The relative differences between the corresponding frequencies, $\left|\omega_{n}^{*}\left(k^{*}\right)-\omega_{n}^{*(f)}\left(k^{*}\right)\right| / \omega_{n}^{*}\left(k^{*}\right)$, where $\omega_{n}^{*}\left(k^{*}\right)$ are for $h^{*}=1 \times 10-4$, are shown in figure 5 . It is seen that the relative differences for $n \leqslant 5$ are small. The corresponding wave profiles are different near the walls of the channel, see figure 15, where the amplitudes of the hydroelastic waves are zero because of the ice clamped conditions on the vertical walls. Figure 5 shows that the relative differences decay with $k^{*}$. However, the absolute differences increase with $k^{*}$, which corresponds to our expectation that a small ice thickness is still important for relatively short waves.

The frequencies of the hydroelastic waves $\omega_{n}^{*}\left(k^{*}\right)$, see section 3 , are expected to converge to the wave frequencies calculated by the rigid-ice model, see section 4 , where the ice thickness increases. The dispersion relations of hydroelastic waves are shown in figure $6(\mathrm{a})$-(e) for $n=1,2,3,4,5$ and ice thickness of $h^{*}=0.2,0.3$ and 0.4 . The frequencies of waves propagating in the channel with rigid ice plates are also shown there. The half-width of the lead is $a^{*}=0.22$ in figure 6 . Note different ranges for the frequencies $\omega_{n}^{*}\left(k^{*}\right)$. The first frequency, $\omega_{1}^{*}\left(k^{*}\right)$ is weakly dependent on the ice thickness and is well approximated by the rigid-ice model, see figure $6(\mathrm{a}), \omega_{1}^{*}\left(k^{*}\right) \approx \omega_{1}^{*(r)}\left(k^{*}\right)$, where $h^{*} \geqslant 0.2$. The frequencies $\omega_{n}^{*}\left(k^{*}\right)$ calculated with $h^{*}=0.2,0.3$ and 0.4 for $n=2,3,4,5$, see figure $6(\mathrm{~b})-(\mathrm{e})$, are close to each other and to the corresponding frequencies $\omega_{n}^{*(r)}\left(k^{*}\right)$ by the rigid-ice model for short waves, where $k^{*}>1$. For long waves, $0<k^{*}<1$, the ice 


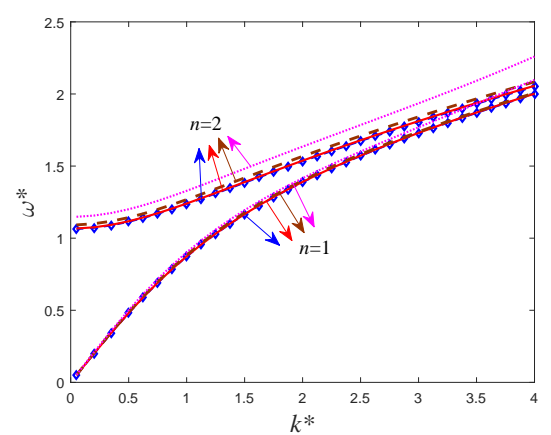

(a)

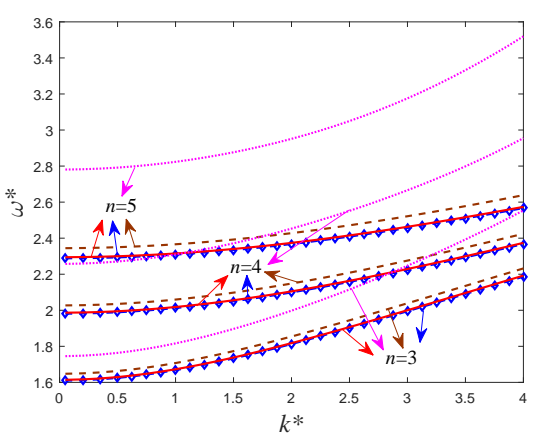

(b)

Figure 4: Dispersion relations for hydroelastic waves in the ice channel with the lead of half-width $a^{*}=0.22$ and the ice thickness $h^{*}=1 \times 10^{-4}$ (solid lines), $1 \times 10^{-3}$ (dashed lines) and $4 \times 10^{-3}$ (dotted lines) for $(a) n=1$ and $n=2,(b)$ $n=3,4,5$. Dispersion relations for the channel without ice cover are shown by rhombus markers.

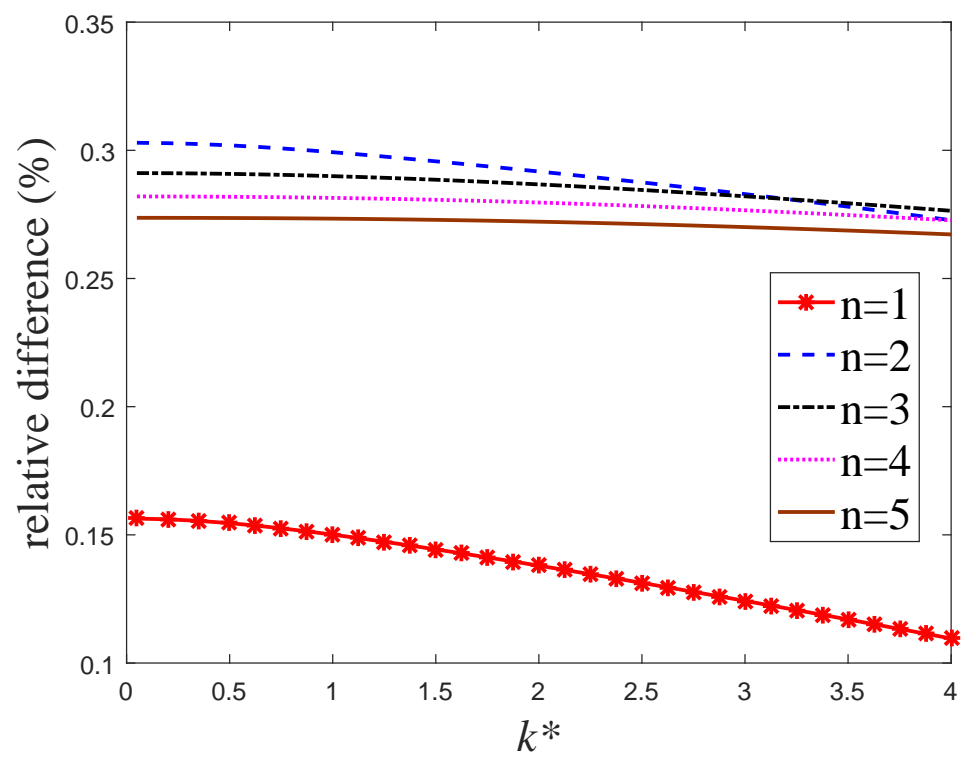

Figure 5: Relative differences between the frequencies $\omega_{n}^{*}\left(k^{*}\right)$ of hydroelastic waves for $h^{*}=1 \times 10^{-4}, a^{*}=0.22$ and the corresponding frequencies $\omega_{n}^{*(f)}(k)$ of the water waves in the same channel without ice for $n=1,2,3,4,5$.

thickness of $h^{*}=0.2$ is not large enough for the rigid-ice model. However, the frequencies $\omega_{n}^{*}\left(k^{*}\right)$ for $h^{*}=0.4$ are well approximated by $\omega_{n}^{*(r)}\left(k^{*}\right)$, where $n=2,3,4,5$. Hydroelastic waves in the ice cover can be modeled within the thin elastic plate approximation (2.1) if the ice thickness $h^{*}$ is much smaller than the wave length $\lambda^{*}=2 \pi / k^{*}$, say $h^{*} / \lambda^{*}<0.1$. This inequality gives $k^{*}<\pi /\left(5 h^{*}\right)$ and $k^{*}<1.57$ for $h^{*}=0.4$. For shorter waves, the thin-plate approximation is not applicable but then the ice cover can be modeled as a rigid plate, see figure 6 . 


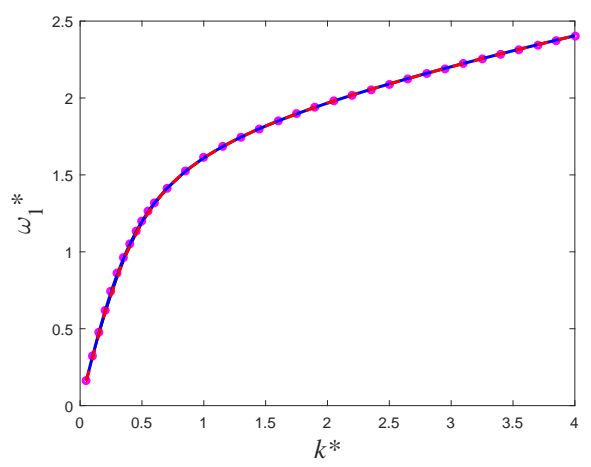

(a)

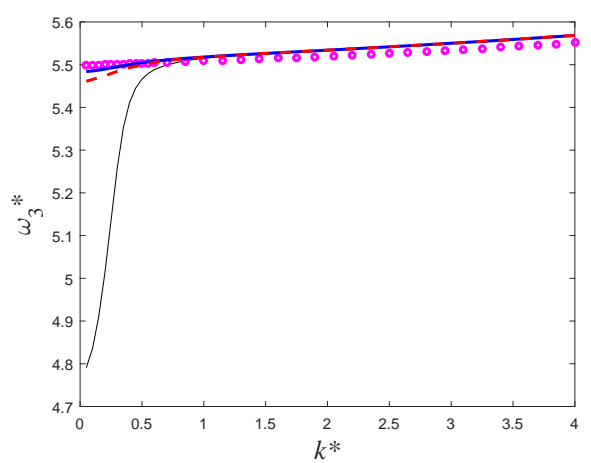

(c)

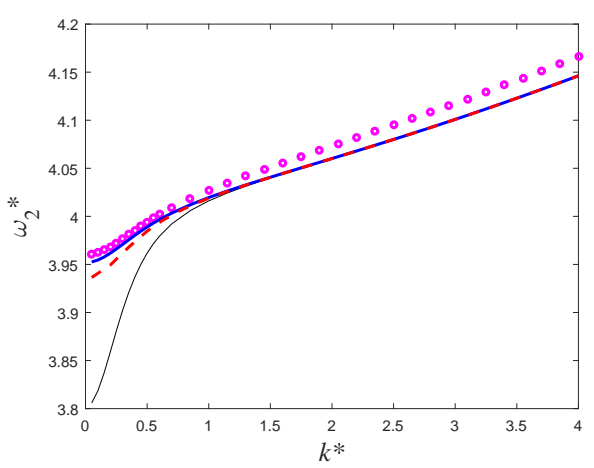

(b)

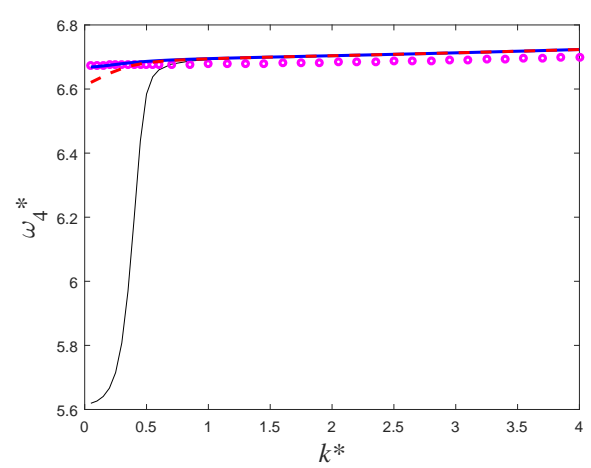

(d)

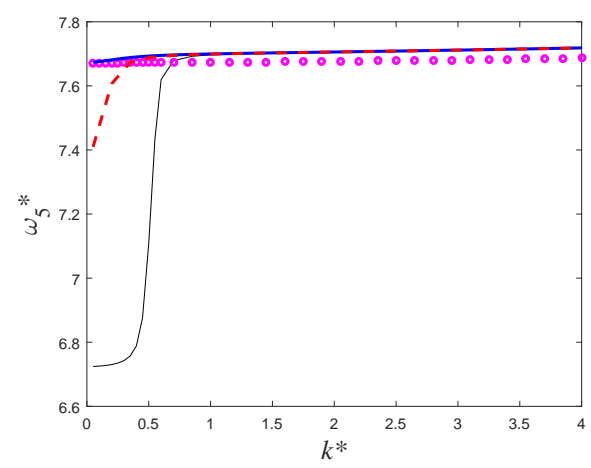

(e)

Figure 6: Dispersion relations for first five hydroelastic waves, $\omega^{*}=\omega_{n}^{*}\left(k^{*}\right)$, and ice thickness $h^{*}=0.2$ (thin solid lines), $h^{*}=0.3$ (dashed lines) and $h^{*}=0.4$ (thick solid lines). The corresponding frequencies by the rigid-ice model are shown by circle markers.

The relative differences between the corresponding frequencies $\left|\omega_{n}^{*}\left(k^{*}\right)-\omega_{n}^{*(r)}\left(k^{*}\right)\right| / \omega_{n}^{*}\left(k^{*}\right)$, where $\omega_{n}^{*}\left(k^{*}\right)$ are calculated for $h^{*}=0.4$, are shown in figure 7 for $n=1,2,3,4,5$. Only for long waves with $k^{*}<0.25$, the relative difference for the lowest frequency $\omega_{1}^{*}\left(k^{*}\right)$ is greater than $1 \%$. Therefore, it is reasonable to conclude that the ice elasticity is less 


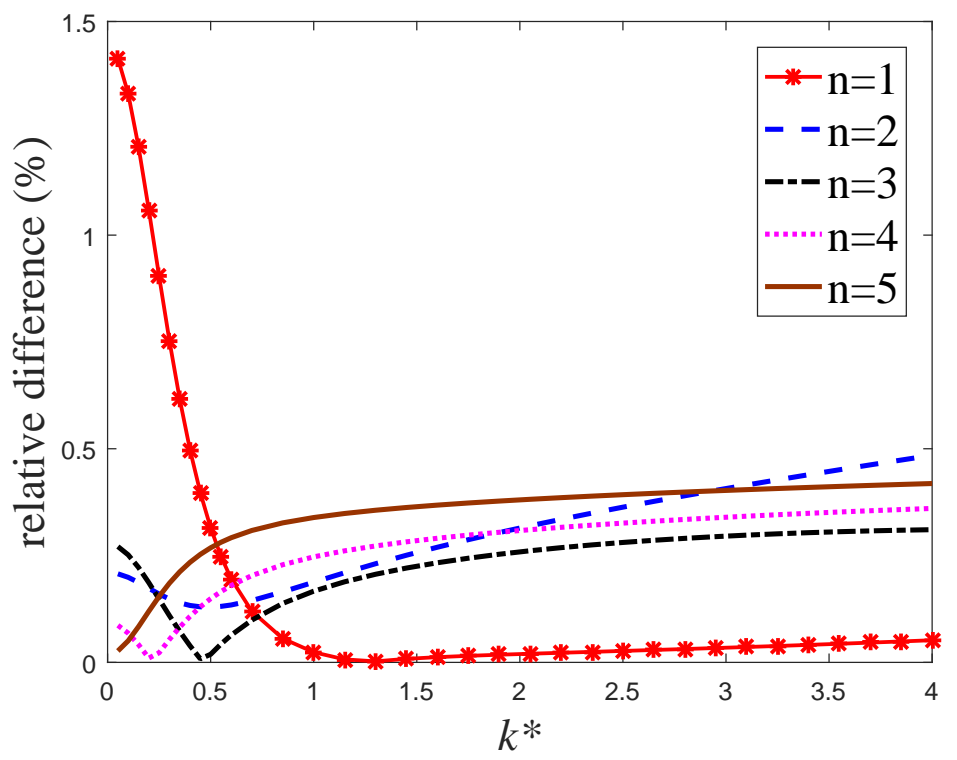

Figure 7: Relative differences between the frequencies $\omega_{n}^{*}\left(k^{*}\right)$ of hydroelastic waves for $h^{*}=0.4$ and the corresponding frequencies $\omega_{n}^{*(r)}\left(k^{*}\right)$ of water waves in the same channel with the lead $a^{*}=0.22$ and rigid ice cover for $n=1,2,3,4,5$.

important for short waves and thick ice. This is illustrated by figure 8 , where the $k^{*}-h^{*}$ plane (wavenumber $k^{*}$ and ice thickness $h^{*}$ ), $0.2<h^{*}<0.4$, is divided for each mode $\omega_{n}^{*}\left(k^{*}\right)$ by a corresponding line into two parts. The relative difference for $\omega_{n}^{*}\left(k^{*}\right)$ is less than $1 \%$ above the corresponding line, see the legend in figure 8 , where the ice elasticity can be neglected. The figure provides that the ice cover can be approximated as rigid for the first five modes if the wave is short enough, $k^{*}>b^{*}-d^{*} \cdot h^{*}$, where $b^{*}=1.2$, $d^{*}=2.5$ and $0.2<h^{*}<0.4$.

The dispersion relations of the first five hydroelastic waves are shown in figure $9(\mathrm{a})$ for ice thickness $h^{*}=0.01$ in both elastic and rigid ice models. It is seen that the ice flexibility is important for long waves, $k^{*}<6$. For short waves with $k^{*}>6$, the ice cover with thickness $h^{*}=0.01$ behaves as a rigid plate. Figure $9(\mathrm{~b})$ shows the dispersion relations for waves propagating in the open water (gravity waves, GW) and in infinite ice sheet (flexural-gravity waves, FGW) together with the dispersion relation of the first hydroelastic wave for the ice thickness $h^{*}=0.01$ and the dimensionless width of the lead $a^{*}=0.22$. The dispersion relations of flexural-gravity waves and gravity waves divide the quarter plane,$k^{*}>0$ and $\omega^{*}>0$, into three regions I, II and III. We can see that the dispersion curve of the first hydroelastic wave starts in the region I at $k^{*}=0$ and $\omega^{*}=0$, where $\omega^{*}>\omega_{F G W}^{*}$, intersects the FGW curve at $k^{*}=2.44$ and $\omega^{*}=1.73$ which is shown by black point in figure $9(\mathrm{~b})$, and then stays in the region II as it was predicted by Marchenko (1997). The first hydroelastic wave, dispersion relation of which is shown by dotted line in figure $9(\mathrm{~b})$, is strongly dependent on both the width of the channel and the width of the lead. However, in terms of interpretation of the regions I and II, the hydroelastic wave with a frequency $\omega^{*}\left(k^{*}\right)$ in region I, where $k^{*}<2.44$, can be considered as the flexural-gravity wave propagating in the ice cover with the dimensionless wavenumber $k^{*}$ in the $x$ - direction along the channel and the dimensionless wavenumber $k_{y}^{*}$ in the $y$ - direction across the channel, where $\omega^{*}\left(k^{*}\right)=$ 


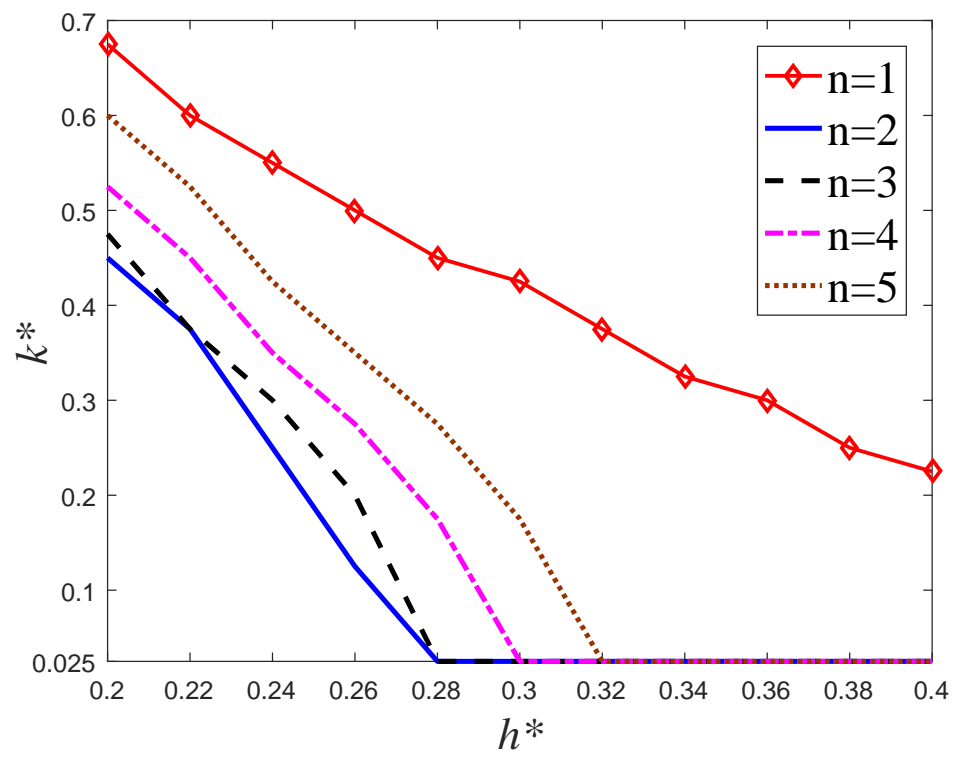

Figure 8: The $k^{*}-h^{*}$ plane with the regions, where ice elasticity can be neglected for waves with numbers $n=1,2,3,4,5$ with relative difference less than $1 \%$ in terms of the corresponding dispersion relation.

$\omega_{F G W}^{*}\left(K^{*}\right)$ and $K^{*}=\sqrt{\left(k^{*}\right)^{2}+\left(k_{y}^{*}\right)^{2}}$. This flexural-gravity wave in the ice plate has the same dimensionless wavenumber $k^{*}$ in the $x$ - direction, as the hydroelastic wave in the channel with the lead, and the real dimensionless wavenumber $k_{y}^{*}$ in the $y$ - direction. This flexural-gravity wave propagates in the $x$ - direction, which is along the channel, and in the $y$ - direction, which is across the channel. Therefore, we can conclude that the long hydroelastic waves in region I can be detected far from the ice plate edge inside the ice sheet. The same arguments applied to region II provides that $k_{y}^{*}$ is imaginary there, which means that such short waves do not penetrate deep in the ice sheet. The hydroelastic waves in region II decay exponentially in the ice cover with the distance from the ice edge. Figure 9(b) shows that the shorter wave, the quicker it decays with the distance. As to the comparison of our hydroelastic wave with gravity waves in both regions I and II, see figure $9(\mathrm{~b})$, the hydroelastic wave can travel both along the channel with the wavenumber $k^{*}$ and across the lead with the real wavenumber $k_{y}^{*}$. The waves from region II, where $k^{*}>2.44$, are localised in the lead.

The dispersion relation of the hydroelastic wave in the channel with the lead (dotted line in figure $9(\mathrm{~b})$ ) and the dispersion relation of the flexural-gravity waves shown by solid line intersect each other at $k^{*}=2.44$, see the black point in figure $9(\mathrm{~b})$. At this point, the wave length, wave frequency and the phase speed of the FGW in the ice and of the first hydroelastic wave in the channel with the lead are equal. However, their group speeds are not equal to each other, see figure $9(\mathrm{c})$. The group speed of the hydroelastic waves in the channel is slightly higher than the group speed of the flexural-gravity waves in ice sheet for $k^{*}<0.21$ and $0.52<k^{*}<1.86$ and smaller for $0.21<k^{*}<0.52$ and $k^{*}>1.86$. The intervals might be affected by accuracy of the numerical calculations of the parameters of the hydroelastic wave. Asymptotic solution of the present problem with a lead as $k^{*} \rightarrow 0$ would be helpful to clarify the difference of the long hydroelastic wave from the FGW. Marchenko (1999) showed that a similar situation occurs for linear hydroelastic waves 
propagating in a semi-infinite ice sheet attached to a vertical wall. In the latter problem, the wave profile is given analytically, which makes it possible to investigate the effect of the vertical wall on the propagating hydroelastic waves in more details. The dispersion relation of the hydroelastic wave in the semi-infinite ice sheet attached to a vertical wall also intersects the dispersion relation of the flexural-gravity waves in the infinite ice sheet at a single point, where the wave length, wave frequency and both the phase and group speeds of the hydroelastic waves in infinite and semi-infinite ice sheets are equal, see Marchenko (1999). This comparison shows that the presence of a lead in the ice channel is important for characteristics of the hydroelastic waves in the channel.

A gravity wave from the lead cannot enter the ice sheet if $k_{y}^{*}$ is much smaller than $k^{*}$, which implies that the angle between the wave propagation and the normal to the ice edge is large. The corresponding analysis has not been done yet. This analysis is expected to provide the conditions, where the ice strains in the hydroelastic waves are relatively large.

\subsection{Phase speeds and critical speeds of hydroelastic waves in the ice channel}

The phase speeds, $c^{*(n)}\left(k^{*}\right)=\omega_{n}^{*}\left(k^{*}\right) / k^{*}$ of the first five hydroelastic waves in the ice channel with the lead, $a^{*}=0.22$, are shown in figure 10(a) for the ice thickness $h^{*}=0.01$. The presence of the lead significantly changes the behaviour of the phase speeds. The phase speeds in the ice channel without a lead decrease with increase of $k^{*}$ for small $k^{*}$, then take their minimum values, which are known as critical speeds, and then increase with increase of $k^{*}$ again, see Korobkin et al. (2014), fig. 6(b). A vessel moving along an ice channel at a critical speed generates ice deflections, which cannot be described within the linear wave theory, see Marchenko (1997). For a weakly nonlinear regime and two-dimensional problems, Parau \& Dias (2002) derived a forced nonlinear Schrodinger equation for the envelope of ice-sheet deflections caused by a load moving at a critical speed. Dinvay et al. (2019) developed a fully dispersive weakly nonlinear model describing flexural-gravity waves in a floating elastic plate, excited by a moving load. For larger amplitudes, both forced and free steady waves were computed by Guyenne \& Parau (2012) using direct numerical simulations with a boundary integral method.

The presence of water in the channel is negligible for relatively short waves, $k^{*}>5$ for $h^{*}=0.01$, see Shishmarev et al. (2016), fig. 15(a). Such waves are elastic but not hydroelastic. The presence of a lead makes the short waves to propagate mainly along the lead as water waves with the ice on sides of the lead being much less involved in the wave motion. As a result, each wave mode for a channel with a lead, except for the first mode, has at least two critical speeds, see figure $10(\mathrm{a})$, where $\mathrm{d} c^{*(n)} / \mathrm{d} k^{*}=0$ and the

phase speed is equal to the group speed, $c_{g}^{*(n)}\left(k^{*}\right)=\mathrm{d} \omega_{n}^{*} / \mathrm{d} k^{*}$. At each corresponding wave number, we have $\mathrm{d}^{2} \omega_{n}^{*} / \mathrm{d} k^{* 2}=k^{*} \mathrm{~d}^{2} c^{*(n)} / \mathrm{d} k^{* 2}$, which is positive if the phase speed is minimum at that $k^{*}$ and negative if the phase speed is maximum at that $k^{*}$. Note that $\frac{1}{2}\left(\omega_{n}^{*}\left(k^{*}\right)\right)^{\prime \prime}$ is the coefficient of the dispersion term in the nonlinear Schrodinger equation describing the weakly nonlinear wave propagating at the critical speed, see Guyenne \& Parau (2012). Dispersion relations with several critical speeds for a wave mode appear, for example, also in the problem of internal waves under ice, see Wang et al. (2014), figure 2(b) for internal (slow) wave mode.

Analysis of the phase speeds is presented in figure 10(b)-(f). Figure 10(b) shows the phase speed of the hydroelastic wave with the lowest frequency $\omega_{1}^{*}\left(k^{*}\right)$, see line 1 . This phase speed decreases monotonically with increase of the wavenumber. Therefore, $\mathrm{d} c^{*(1)} / \mathrm{d} k^{*}<0$ for $k^{*}>0$ and $\mathrm{d} c^{*(1)} / \mathrm{d} k^{*}=0$ only at $k^{*}=0$. The critical speed of this hydroelastic wave, $c^{*(1)}(0)$, is equal to 1.1 , which is slightly higher than the 


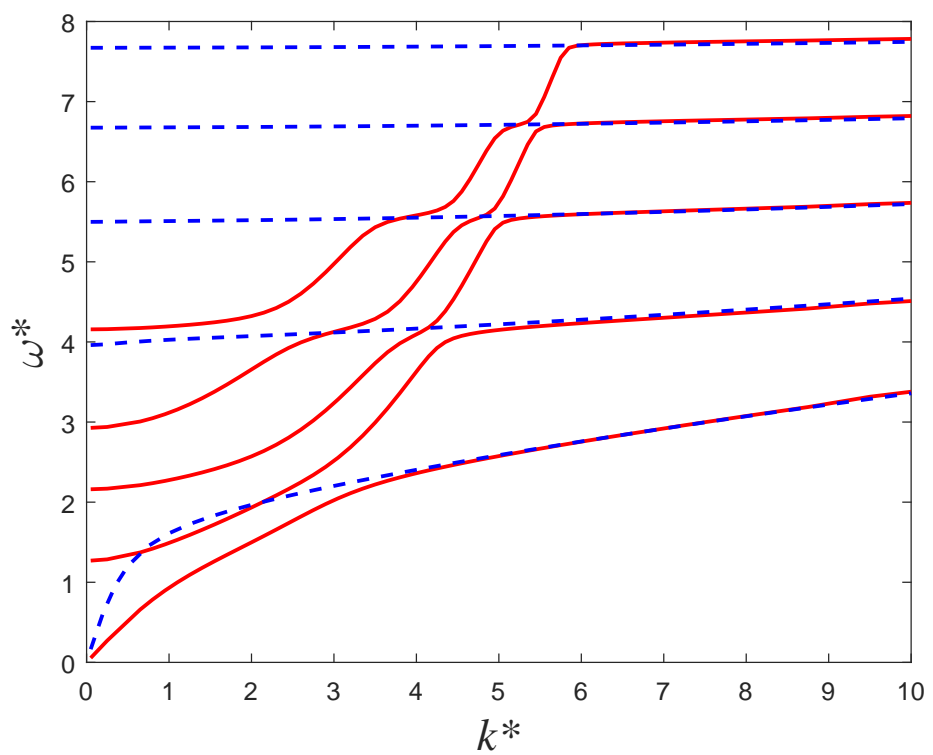

(a)

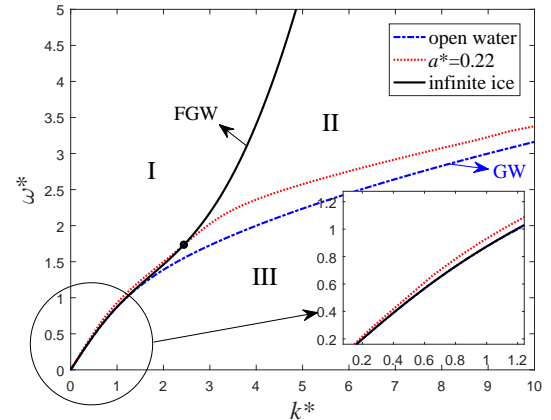

(b)

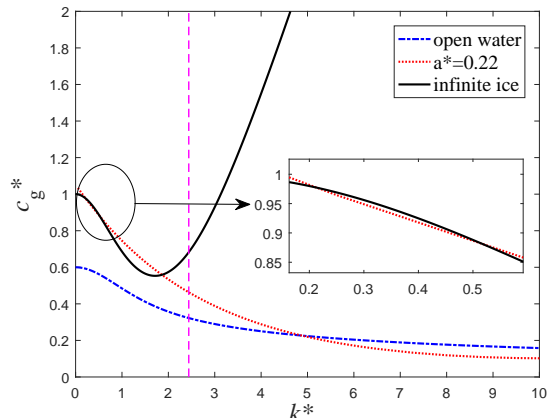

(c)

Figure 9: (a) Dispersion relations for the first five waves, $n=1,2,3,4,5$, $a^{*}=0.22$, calculated within the elastic-ice model with ice thickness of $h^{*}=0.01$ (solid lines) and within the rigid-ice model (dashed lines). (b) Dispersion relations for waves propagating in open water, infinite ice sheet and for the first hydroelastic wave in ice channel with $a^{*}=0.22$. (c) Group speeds for waves propagating in open water, infinite ice sheet and for the first hydroelastic wave in ice channel with $a^{*}=0.22$.

corresponding critical speed of long water waves in the same channel but without ice, $c_{f}^{*(1)}(0)=1$, see line 3 . The curve 4 is also for water waves but in the channel of reduced width equal to the width of the lead $2 a^{*}$. Figure 10(b) shows that the curve 3 and curve 4 are identical, because the first phase speed of waves in channel without ice, $c_{f}^{*(1)}\left(k^{*}\right)=\omega_{1}^{*(f)}\left(k^{*}\right) / k^{*}=\sqrt{\tanh \left(k^{*}\right) / k^{*}}$, depends only on the non-dimensional wavenumber $k^{*}$ but not on the channel width. The phase speed by the curve 2 is for the same channel and the lead but within the rigid-ice model. Figure 10(b) shows that the phase speed of the first hydroelastic wave can be roughly approximated by the phase 


$\begin{array}{lrrr} & k^{*}=1 & k^{*}=2 & k^{*}=3 \\ \omega_{1}^{*(r)} & -3.36 & -3.13 & -2.95 \\ \omega_{2}^{*(r)} & 1.63 & 1.60 & 1.59 \\ \omega_{3}^{*(r)} & 1.13 & 1.13 & 1.12 \\ \omega_{4}^{*(r)} & 0.83 & 0.83 & 0.83 \\ \omega_{5}^{*(r)} & 0.60 & 0.60 & 0.60\end{array}$

Table 1: The dimensionless velocity of the flow across the channel, $\frac{\partial \Phi^{*}}{\partial y^{*}}\left(a^{*}, z^{*}\right)$, at the ice edge, $z^{*}=0$, for different wavenumbers and different wave modes within the rigid ice model.

speed of the one-dimensional water wave. The rigid-ice model predicts much greater phase speed for long waves, $k^{*}<3.5$, where the flow between the lead region, $\left|y^{*}\right|<a^{*}$, and the region under the rigid ice, $a^{*}<\left|y^{*}\right|<L^{*}$, is significant. For shorter wave the flow between these regions is weak and all four phase speeds are close to each other.

For higher wave modes, $n=2,3,4,5$ in figure 10 (c)-(f), the presence of ice plates of thickness $h^{*}=0.01$ in the channel is important, which can be concluded by comparing the phase speeds presented by the curves 1 and 3 . The curves 1,2 and 4 are very close to each other for $k^{*}>5$, see figure 10(c)-(f), which shows that the computed waves are gravity waves confined to the lead region. However, the fluid under the ice sheets is not at rest even for the rigid ice model. From equations (4.6) and (4.11), we find

$$
\frac{\partial \Phi}{\partial y}\left(a^{*}, z^{*}\right)=\sum_{m=0}^{\infty} \frac{-\cosh \left[\left(æ_{m} H\right)\left(z^{*}+1\right)\right]\left(\lambda_{m} H\right)}{\left(æ_{m} H\right) \sinh \left(æ_{m} H\right)} \sin \left(\left(\lambda_{m} H\right) a^{*}\right) \sum_{k=0}^{\infty} F_{k} S_{k m},
$$

at the ice edge, $y^{*}=a^{*}$ for the rigid ice model. The dimonlesionless numerical values of $\frac{\partial \Phi^{*}}{\partial y^{*}}\left(a^{*}, z^{*}\right)$ at $z^{*}=0$ for $k^{*}=1,2,3$ are shown in table 1 . We can find that the values $\frac{\partial \Phi^{*}}{\partial y^{*}}\left(a^{*}, 0\right)$ are not equal to zero for different wave modes and wavenumbers, which indicates that rigid ice model is not equivalent to the channel without ice with the reduced width $2 a^{*}$. However, it should be noted that the absolute values of $\frac{\partial \Phi^{*}}{\partial y^{*}}\left(a^{*}, 0\right)$ decrease with the wavenumber increase. The most notable feature of long waves in figure 10(c)-(f) is the presence of several critical speeds for each mode. We count two critical speeds for $n=2,3$ and four critical speeds for $n=4,5$.

To investigate the influence of ice thickness on the phase speeds, the first and second phase speeds for different ice thicknesses are shown in figure 11. For a given wave number, we find that the phase speeds increase with increase of the ice thickness for $c^{*(1)}\left(k^{*}\right)$ and $c^{*(2)}\left(k^{*}\right)$. We observe from figure 11 (a) that there is no point with $\mathrm{d} c^{*(1)} / \mathrm{d} k^{*}=0$ for $k^{*}>0$. We also observe from figure $11(\mathrm{~b})$ that there are two points with $\mathrm{d} c^{*(2)} / \mathrm{d} k^{*}=0$ for $h^{*}=0.01, h^{*}=0.02, h^{*}=0.03$ and $h^{*}=0.04$. The critical speeds increase with increase of the ice thickness. However, for ice thinkness of $h^{*}=0.06$ there are no critical speeds for the second hydroelastic wave. The critical speeds of the second hydroelastic wave, $c_{c r, 1}^{*(2)}$ and $c_{c r, 2}^{*(2)}$, as functions of the ice thickness $h^{*}$ for the ice channel with the lead half-width $a^{*}=0.22$ are shown in figure 12. The first critical speed of the second hydroelastic wave, $c_{c r, 1}^{*(2)}$ is always smaller than the second critical speed, $c_{c r, 2}^{*(2)}$, see also figure 11(b).

As to the influence of the width of the open water lead on the phase speeds, figure 13 provides the phase speeds of the first and second hydroelastic waves for $h^{*}=0.01$ and different half-width of the open water lead. It is seen that there is one critical speed for 


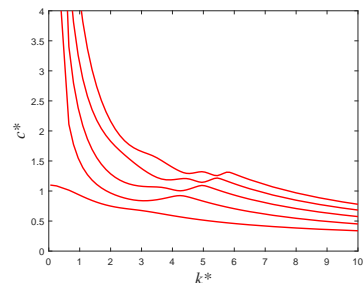

(a)

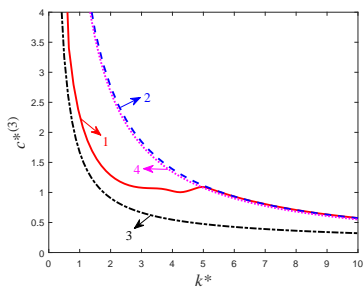

(d)

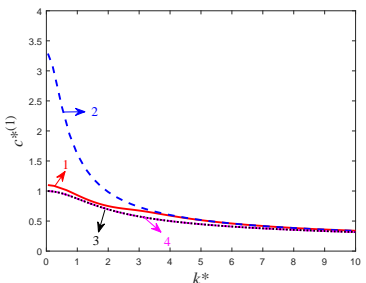

(b)

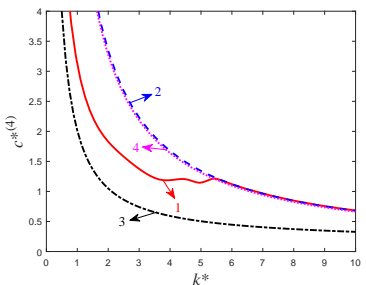

(e)

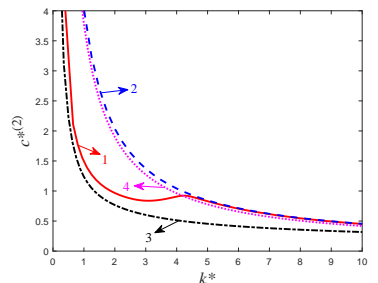

(c)

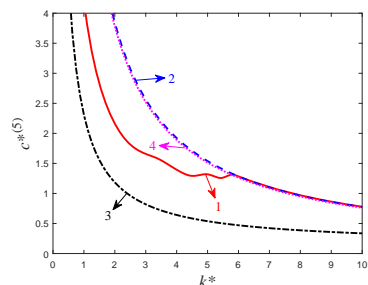

(f)

Figure 10: Phase speeds of hydroelastic waves with $n=1,2,3,4,5$ as functions of the wave number. (a) Phase speeds for ice thickness $h^{*}=0.01$, where $c^{*(n+1)}\left(k^{*}\right)>c^{*(n)}\left(k^{*}\right)$ for $n \geqslant 1$ and $k^{*} \geqslant 0 .(b)-(f)$ Phase speeds $c^{*(n)}\left(k^{*}\right)$ of the hydroelastic waves for $h^{*}=0.01$ (solid lines, 1 ), waves within the rigid-ice model (dashed lines, 2), waves in the channel without ice (dashed-dotted lines, 3 ), and waves in the channel without ice with the reduced width $2 a^{*}$ (dotted lines, 4).

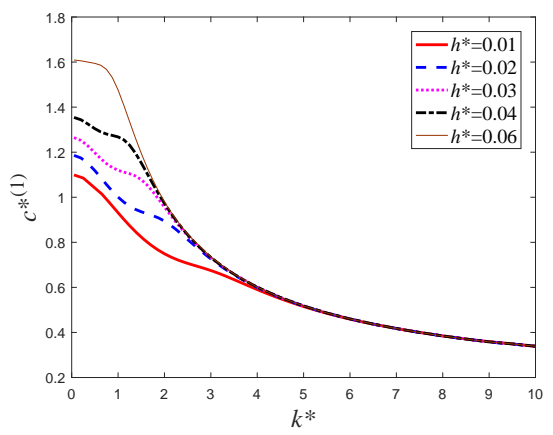

(a)

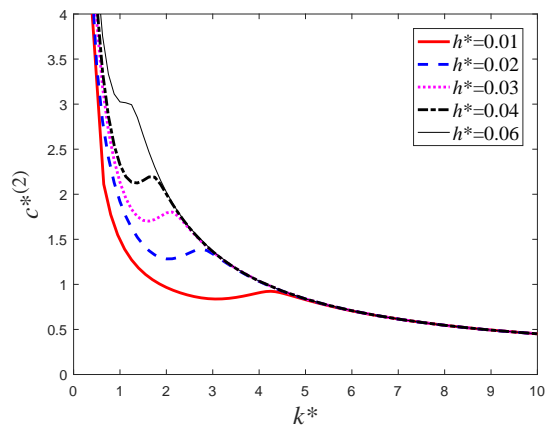

(b)

Figure 11: Phase speeds $c^{*(n)}\left(k^{*}\right)$ for $(a) n=1$ and $(b) n=2$ as functions of the wave number for ice thickness $h^{*}=0.01$ (thick solid lines), 0.02 (dashed lines), 0.03 (dotted lines), 0.04 (dashed-dotted lines) and 0.06 (thin solid lines).

each wave without the lead of open water, $a^{*}=0$. However, for $a^{*}=0.11,0.22,0.44$, 0.8 and 1.2 , there are no critical speed for the first hydroelastic wave, and two critical speeds for the second hydroelastic wave. It is worth further investigating the effect of the width of the open water lead on critical speeds. Figure 14 provides the critical speeds as functions of the half-width of the open-water lead $a^{*}$ for the first and second hydroelastic waves with ice thickness of $h^{*}=0.01$. Note that we do not count here the critical speed of the long first wave at $k^{*}=0$, which exists for any $a^{*}$. It is seen that there is no critical 


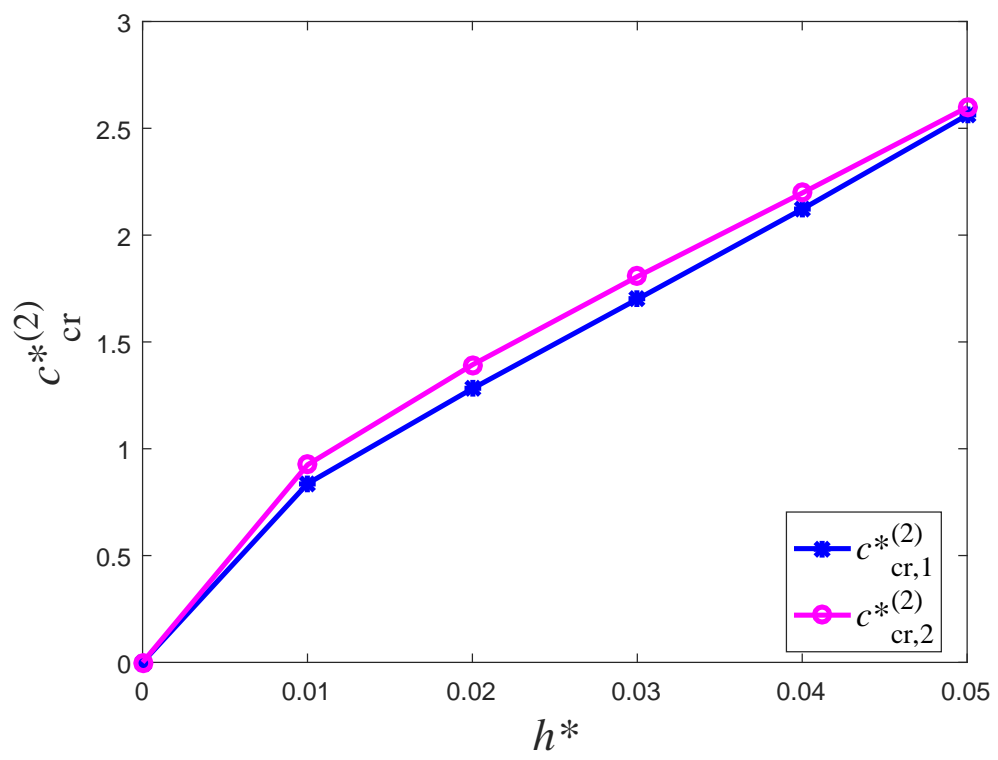

Figure 12: Critical speeds of the second hydroelastic wave, $c_{c r, 1}^{*(2)}$ (lines with asterisk markers) and $c_{c r, 2}^{*(2)}$ (lines with circle markers), as functions of the ice thickness for $a^{*}=0.22$.

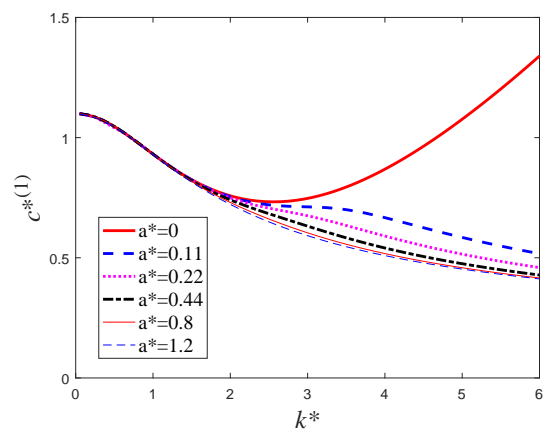

(a)

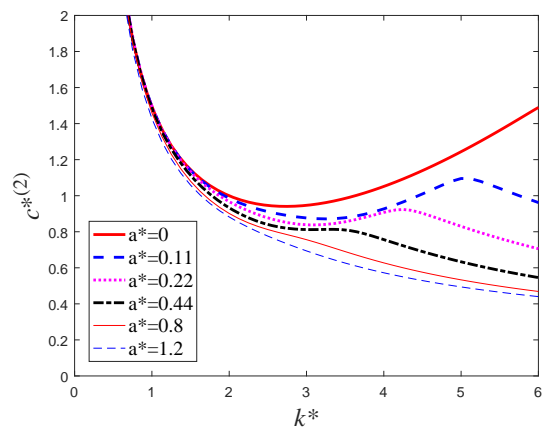

(b)

Figure 13: Phase speeds $c^{*(n)}\left(k^{*}\right)$, for $(a) n=1,(b) n=2$ and ice thickness $h^{*}=0.01$ as functions of the wave number without the lead $\left(a^{*}=0\right.$, thick solid lines), and with the lead of half-width $a^{*}=0.11$ (thick dashed lines), $a^{*}=0.22$ (dotted lines), $a^{*}=0.44$ (dashed-dotted lines), $a^{*}=0.8$ (thin solid lines), and $a^{*}=1.2$ (thin dashed lines).

speed for the first hydroelastic wave with $a^{*}>0.1$. For $0.02 \leqslant a^{*}<0.1$, there are two critical speeds for the first hydroelastic wave. For the second hydroelastic wave, there is one critical speed for $0<a^{*}<0.04$, there are two critical speeds for $0.04 \leqslant a^{*}<0.46$, then the critical speeds merge at $a^{*} \approx 0.46$ and there are no critical speeds for $a^{*}>0.46$. The critical speeds decrease with increase of the width of the lead. 


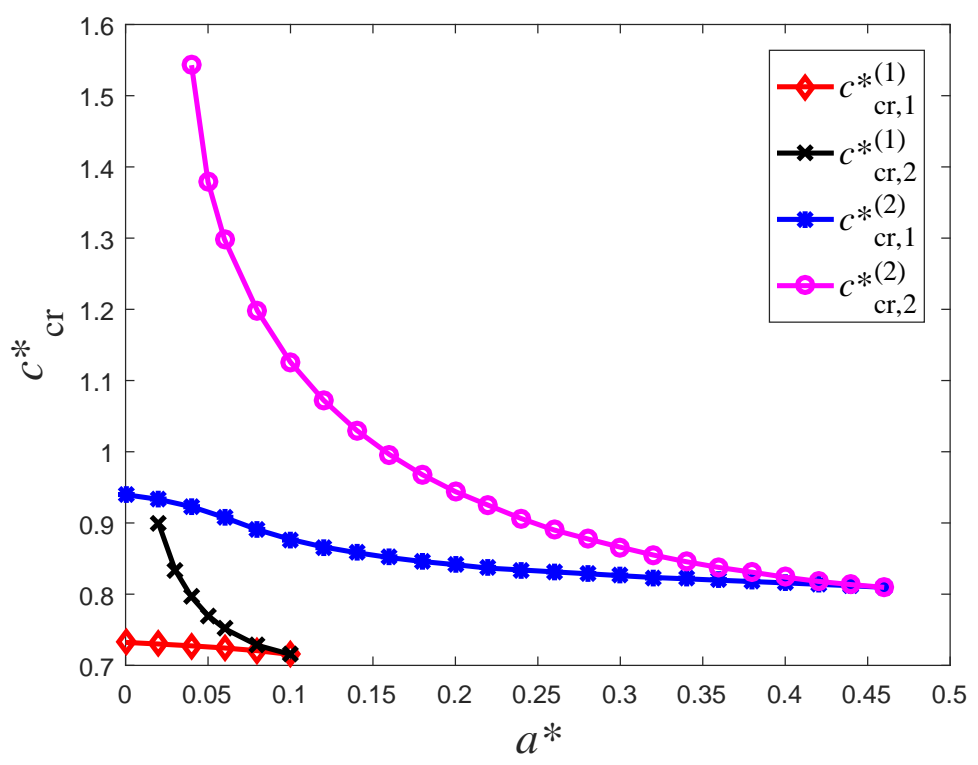

Figure 14: Critical speeds of the first and second hydroelastic waves as functions of the lead half-width $a^{*}$ for ice thickness $h^{*}=0.01$.

\subsection{Profiles of hydroelastic waves}

Linear hydroelastic waves propagating in an ice channel with a lead are sinusoidal along the channel, see $(2.6)$, with the wave length $2 \pi / k^{*}$. For example, a wave with $k^{*}=0.5$ is of length $\lambda^{*}=12.6$, which is greater than the channel width, $2 L^{*}=4.8$, the channel depth, $H^{*}=1$, and the width of the symmetric lead, $2 a^{*}=0.44$, in the main part of our calculations. The profiles of the waves across the channel are more complicated. The profile of the $n$-th hydroelastic wave is described by the even function $F_{n}^{*}\left(y^{*}\right)$, where $\left|y^{*}\right|<L^{*}$, which is calculated as part of the solution. The functions $F_{n}^{*}\left(y^{*}\right)$ are normalised in such a way that their maximum magnitudes are equal to 1 . The wave profiles $F_{n}^{*}\left(y^{*}\right)$ depend on the parameters of the channel and the ice plates, as well as on the wave number $k^{*}$. Only waves symmetric with respect to the centreline of the channel are considered in this study.

The computed profiles $F_{n}^{*}\left(y^{*}\right)$ of the hydroelastic waves across the channel are shown in figure 15 for $n=1,2,3,4,5$ and small ice thickness of $h^{*}=1 \times 10^{-4}$. The half-width of the open water lead is $a^{*}=0.22$ and $k^{*}=0.5$. The wave profiles for the same channel without ice, $F_{n}^{*(f)}\left(y^{*}\right)=\cos \left(\frac{(n-1) \pi}{L^{*}} y^{*}\right)$, are also presented for comparison. Only half, $0<y^{*}<L^{*}$, of the channel width is shown because of the symmetry of the computed waves. One can see that the deflection of ice and elevation of the open water are close to each other at the ice edge, $y^{*}=a^{*}$, for $n=1,2,3,4$ and 5 , but not equal even for this small thickness of the ice. The wave profiles for the ice channel and the same channel without ice are close to each other except near the wall, $y^{*}=L^{*}$, where the ice plate is clamped to the wall, $F\left(L^{*}\right)=F^{\prime}\left(L^{*}\right)=0$. Figure 15 (a) shows that the lowest hydroelastic wave is of constant elevation inside the channel but changes significantly near the wall. This indicates high bending stresses near the wall for this wave.

The hydroelastic wave profiles $F_{n}^{*}\left(y^{*}\right)$ for $n=1,2,3,4,5$ and ice thickness $h^{*}=0.4$ are shown in figure 16 . The computed deflections of the ice were multiplied by 100 in this 


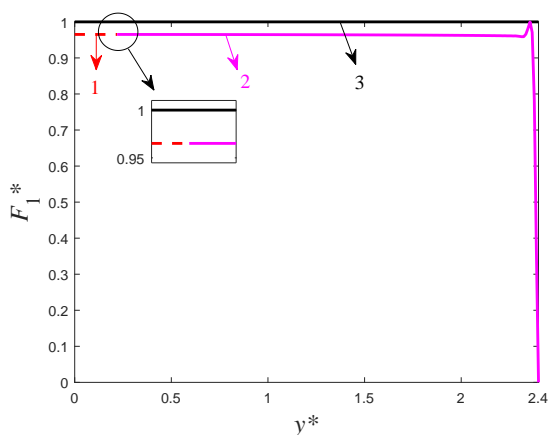

(a)

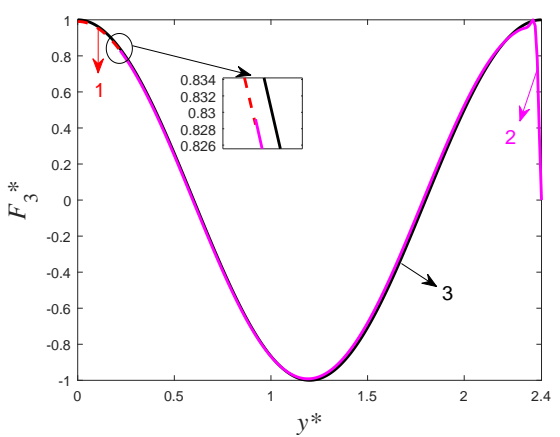

(c)

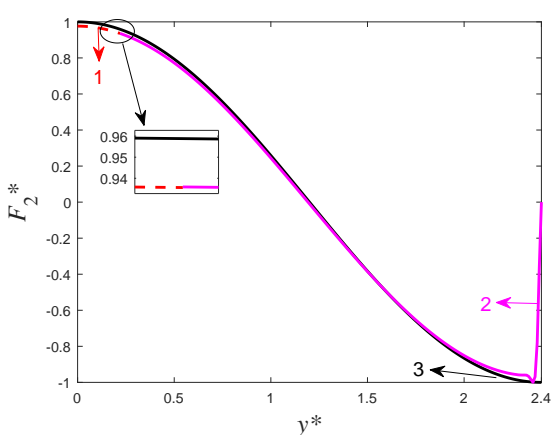

(b)

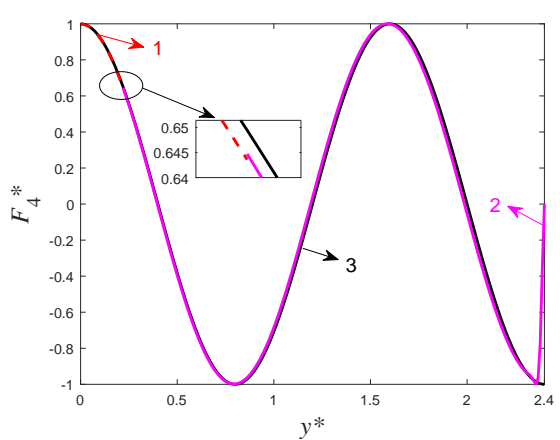

(d)

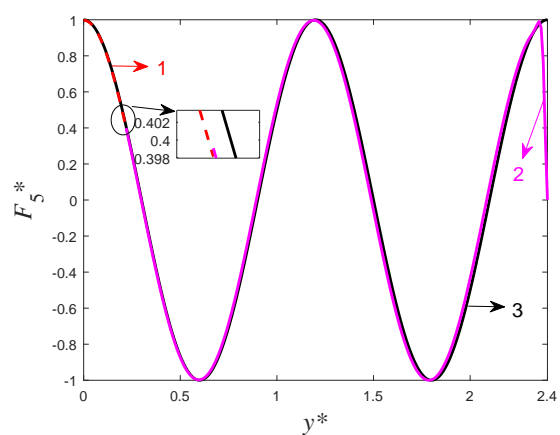

(e)

Figure 15: Profiles $F_{n}^{*}\left(y^{*}\right)$ of the hydroelastic waves (solid lines) for $k^{*}=0.5$ in the ice channel with a lead. Half-width of the lead $a^{*}=0.22$. Ice thickness $h^{*}=1 \times 10^{-4}$. The corresponding water wave profiles for the same channel without ice are shown by lines 3 .

figure. It is seen that the ice deflection is very small compared to the water elevation in the lead.

The wave profiles $F_{n}^{*}\left(y^{*}\right)$ for $n=1,2,3,4,5$ and ice thickness $h^{*}=0.01$ are shown in figure 17. The functions $F_{n}^{*}\left(y^{*}\right)$ are defined by the series (3.1) with respect to the modes $\psi_{m}\left(y^{*}\right), m \geqslant 1$, with the coefficients $F_{n, m}$ computed as it is explained in section 5.1. The non-dimensionalised modes are depicted in figure 21. Comparing figures 17 and 21, we 


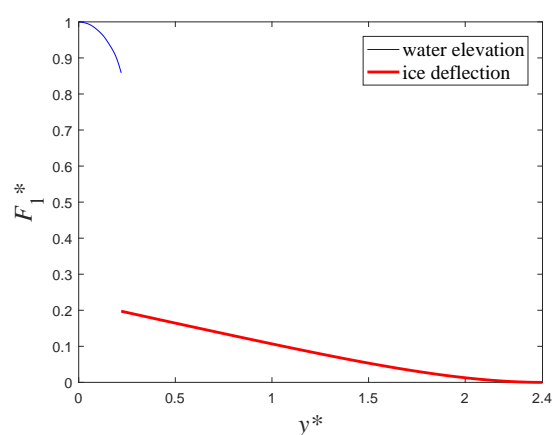

(a)

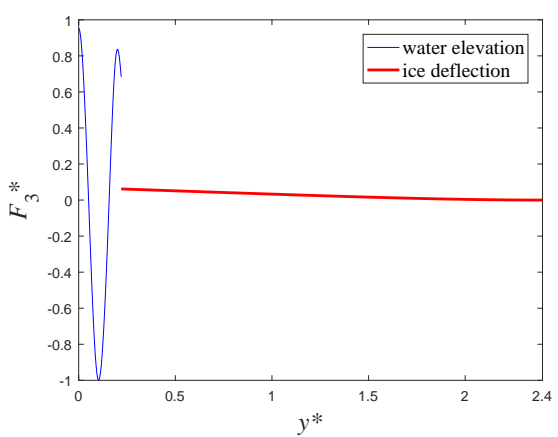

(c)

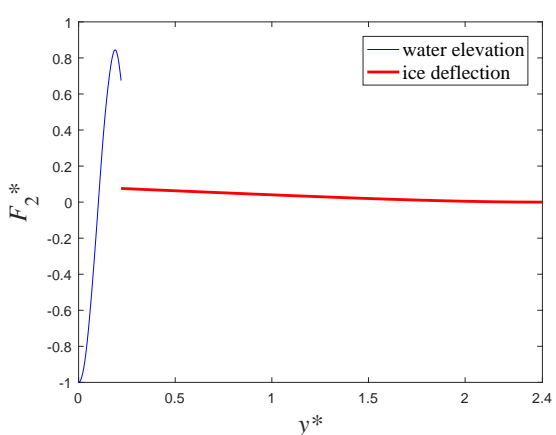

(b)

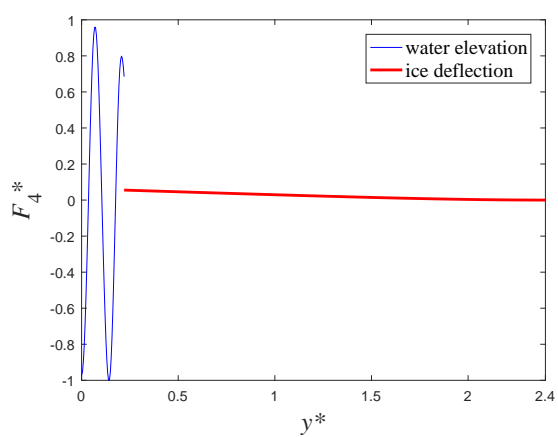

(d)

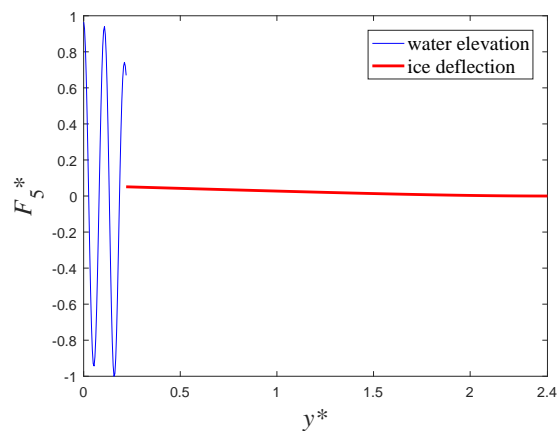

(e)

Figure 16: Profiles $F_{n}^{*}\left(y^{*}\right)$ of the hydroelastic waves for $k^{*}=0.5$ in the ice channel with a lead. Half-width of the lead $a^{*}=0.22$. Ice thicknessis is $h^{*}=0.4$. The deflections of ice were multiplied by 100 .

can conclude that the main contribution to the $n$-th wave profile $F_{n}^{*}\left(y^{*}\right)$ comes from the mode $\psi_{m}\left(y^{*}\right)$, where $m \leqslant n$. In particular, the coefficients of the third wave profile, $F_{3, m}$, which are greater by module than $10^{-3}$ are shown in table 2 . From the first equation of (2.6), we can easily obtained the three-dimensional wave, $w_{n}^{*}=F_{n}^{*}\left(y^{*}\right) \cos \left(k x-\omega_{n} t\right)$ of waves propagating in the ice channel with a lead of open water. The three-dimensional wave profiles for $n=3$, and $t=0$ are also shown in figure 18 . We can clearly see the 


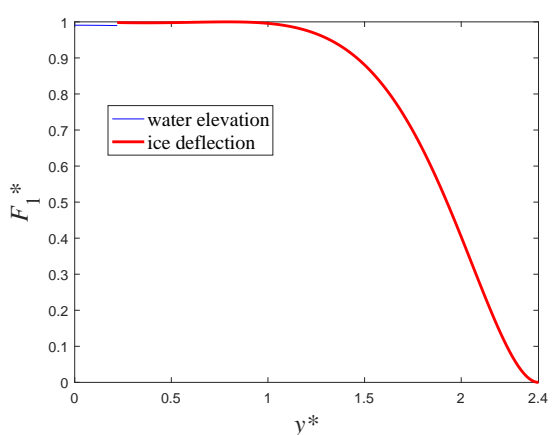

(a)

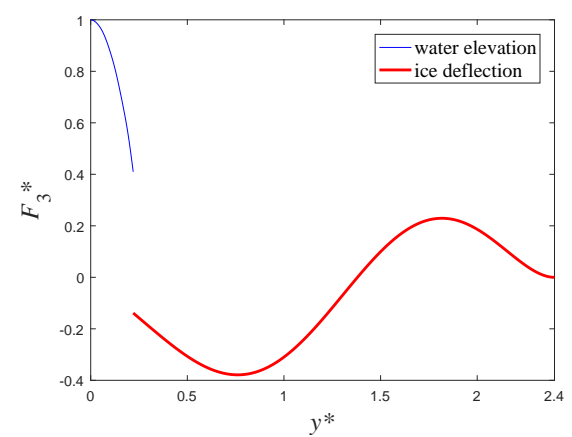

(c)

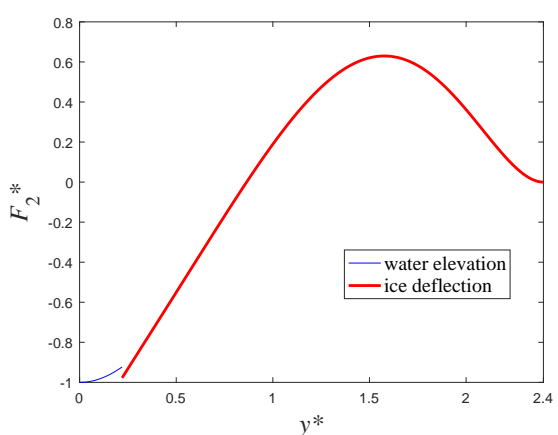

(b)

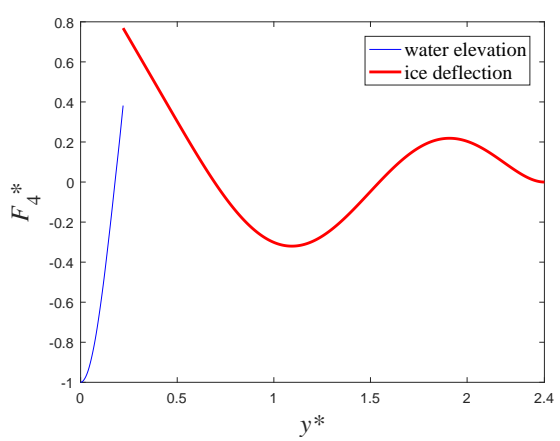

(d)

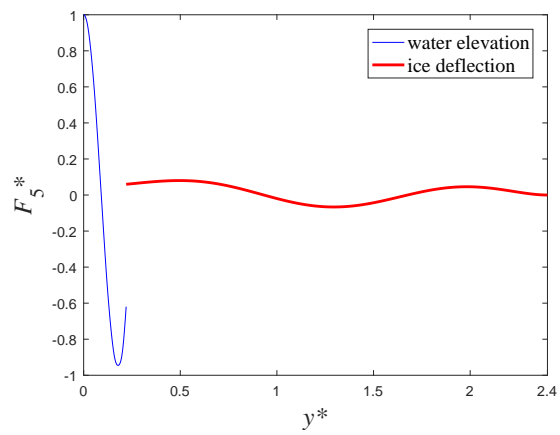

(e)

Figure 17: Profiles $F_{n}^{*}\left(y^{*}\right)$ of the hydroelastic waves for $k^{*}=0.5, a^{*}=0.22$ and ice thickness $h^{*}=0.01$.

difference between ice deformation and water elevation, as shown in figure 17c. The wave changes periodically in the $x$ direction.

The strain distribution in the ice sheet is an important characteristic indicating potential breaking of ice caused by ship or wind generated waves. The maximum strain at each point of the ice surface can be obtained using the eigenvalues of the strain tensor,

$$
\epsilon= \pm \frac{h}{2}\left(\begin{array}{ll}
w_{x x} & w_{x y} \\
w_{x y} & w_{y y}
\end{array}\right)
$$




$\begin{array}{lrlr}F_{3,1} & 8.3910 & F_{3,8} & -0.0136 \\ F_{3,2} & -2.2179 & F_{3,9} & -0.0103 \\ F_{3,3} & -7.2201 & F_{3,10} & -0.0044 \\ F_{3,4} & -0.3904 & F_{3,11} & -0.0035 \\ F_{3,5} & -0.2600 & F_{3,12} & -0.0018 \\ F_{3,6} & -0.0570 & F_{3,13} & -0.0015 \\ F_{3,7} & -0.0401 & & \end{array}$

Table 2: The values of $F_{3, m}$ greater than $10^{-3}$

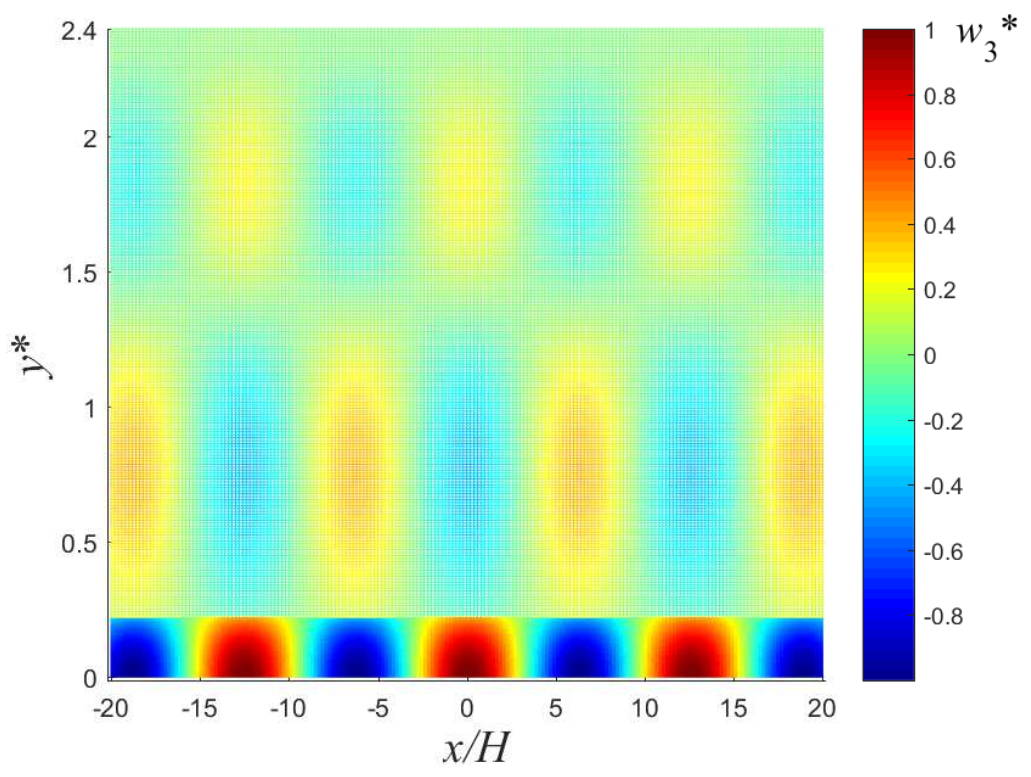

Figure 18: Three dimensional wave for the third mode with $a^{*}=0.22$ and ice thickness $h^{*}=0.01$.

where $h / 2$ and $-h / 2$ are for the lower and upper surface of the ice plate, respectively. To determine the maximum strain as a function of $y$ across the channel for each wave mode, we calculate four following functions of $y$ (Batyaev \& Khabakhpasheva (2015))

$$
\left\{\begin{array}{c}
\epsilon_{\max , 1}(y)=\frac{h}{2}\left|F^{\prime \prime}(y)\right|, \quad \epsilon_{\max , 2}(y)=\frac{h}{2} k^{2}|F(y)| \\
\epsilon_{\max , 3}(y)=\frac{h}{2} k F^{\prime}(y) \sqrt{\frac{4 k^{2} F^{\prime \prime}(y) F(y)-4 k^{2} F^{\prime}(y)^{2}}{\left(F^{\prime \prime}(y)+k^{2} F(y)\right)^{2}-4 k^{2} F^{\prime}(y)^{2}}} \\
\epsilon_{\max , 4}(y)=\frac{h}{2} F^{\prime}(y) \frac{F^{\prime \prime}(y)^{2}+k^{4} F(y)^{2}-2 k^{2} F^{\prime}(y)^{2}}{\sqrt{\left(F^{\prime \prime}(y) F(y)-F^{\prime}(y)^{2}\right)\left[\left(F^{\prime \prime}(y)+k^{2} F(y)\right)^{2}-4 k^{2} F^{\prime}(y)^{2}\right]}}
\end{array}\right.
$$

where $F(y)$ is the wave profile of a wave across the channel with the maximum magnitude $1 \mathrm{~m}$, see section 5.1. Note that $\epsilon_{\max , 3}(y)$ and $\epsilon_{\max , 4}(y)$ exist only there where $\left[\left(F^{\prime \prime}(y)+\right.\right.$ $\left.\left.k^{2} F(y)\right)^{2}-4 k^{2} F^{\prime}(y)^{2}\right]^{2} \geqslant\left(F^{\prime \prime}(y)^{2}-k^{4} F(y)^{2}\right)^{2}$. The maximum strain $\epsilon_{\max }(y)$ at each $y$ is 
the maximum of the following four values $\epsilon_{\max , 1}(y), \epsilon_{\max , 2}(y), \epsilon_{\max , 3}(y)$, and $\epsilon_{\max , 4}(y)$. In figure $19, \epsilon_{\max }(y)$ is shown for the first five hydroelastic waves where $k^{*}=0.5, a^{*}=0.22$ and $h^{*}=0.01$. It is seen that the distributions $\epsilon_{\max }(y)$ peak at the walls, $y^{*}=2.4$, for $n=1,2,3,4,5$. The strains near the free edge of the ice sheet are small, compare with figure 17. For the conditions of the hydroelastic waves in this figure, cracks are expected along the walls if the strain there is greater than the so-called yield strain $\epsilon_{Y}$. The yield strain for ice is estimated as $8 \times 10^{-5}$ (Brocklehurst et al. 2011). Figure 19(a) provides that the strains in the ice plate caused by the lowest hydroelastic wave are below the yield strain for ice if the magnitude of this wave is smaller than $8 \mathrm{~mm}$. These cracks along the walls may break the connection between the ice sheet and the walls without breaking the ice sheet into pieces.

\section{Conclusions}

The effect of a lead of open water on the hydroelastic waves propagating in an ice channel was studied within the linear theory of hydroelasticity. The normal mode method and the eigenfunction expansion method were used to determine the dispersion relations and the profiles of these waves for elastic ice and rigid ice models, respectively. Only waves symmetric with respect to the centerplane of the channel were considered. It was shown that the presence of a lead significantly changes the waves propagating along the ice channel. In a channel completely covered with an ice plate, short waves propagate through the ice with water in the channel being less involved in the motion. These waves are elastic waves. Very long waves in an ice channel depend on the presence of ice only in a closed proximity of the walls of the channel, where the ice plate is clamped to the walls. However, the dispersion relations are affected by the ice cover even for very long waves, if the ice thickness is moderate. Hydroelastic waves are in between very long waves, which are gravity dominated, and short waves, which are dominated by the ice elasticity. The interplay between gravity and elastic effects makes these waves particular with the most prominent their feature being the existence of a single so-called critical speed for each mode of the waves in the ice channel.

The presence of a lead in the ice channel changes the short waves. They are gravity waves now. They are confined to the part of the channel under the lead with ice deflections being negligible. Moreover, the flow between the region under the ice and the region under the lead becomes negligible for higher modes of the waves. The lead also modifies the hydroelastic waves, which are long enough for both gravity and elastic effects being equally important. The most important modification is related to the critical speeds of these waves. Each wave mode starting from the lowest one can have zero, one, two or even more critical speeds depending on the relative width of the lead. At some particular conditions two critical speeds may merge providing a point on the dispersion curve, where both the first and second derivatives of the phase speed with respect to the wavenumber are zero. At that point, the phase speed is equal to the group speed and the second derivative of the wave frequency as a function of the wave number is zero. Waves propagating at a critical speeds cannot be linear. They are described by the nonlinear Schrodinger equation for small amplitudes and can be computed for moderate wave amplitudes. The second derivative of the wave frequency, $\frac{1}{2}\left(\omega_{n}^{*}\left(k^{*}\right)\right)^{\prime \prime}$, appears as the coefficient of the dispersion term in the nonlinear Schrodinger equation. If two critical speeds merge, this coefficient becomes zero, which would require revision of the weakly nonlinear hydroelastic waves in such special conditions. In our analysis, we did not find conditions, where three or more critical speeds merge, however, potentially this is possible making the theory of weakly nonlinear hydroelastic waves even more challenging. 


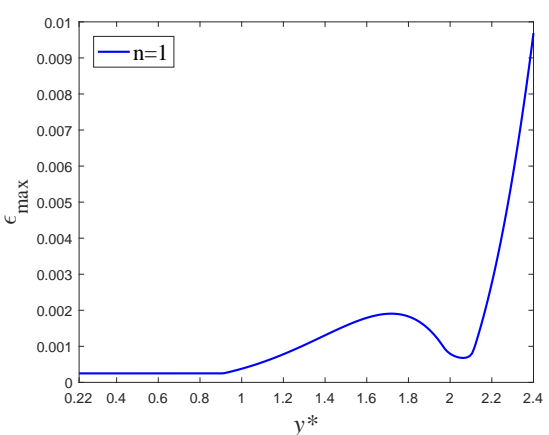

(a)

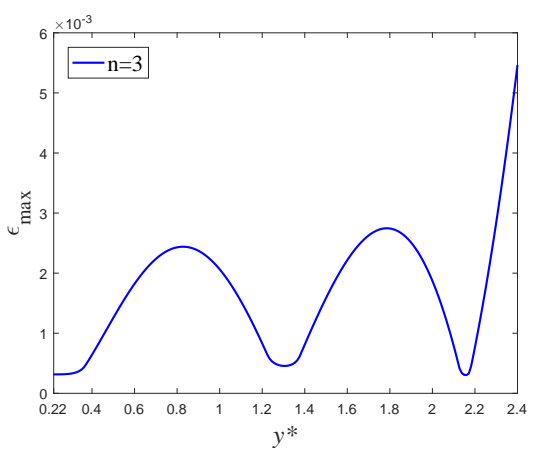

(c)

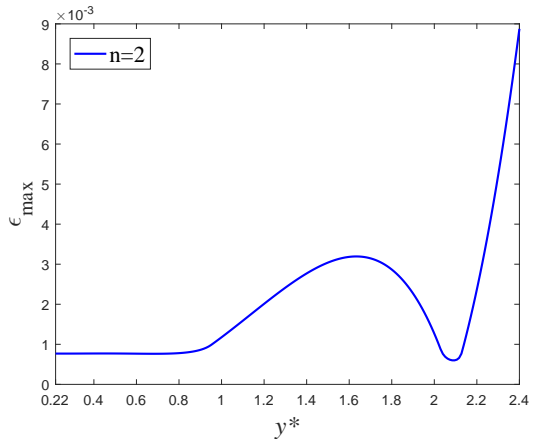

(b)

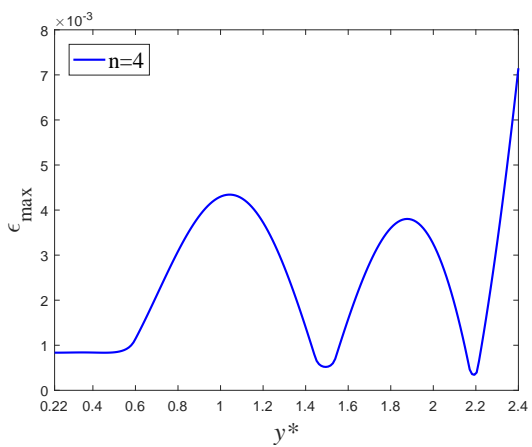

(d)

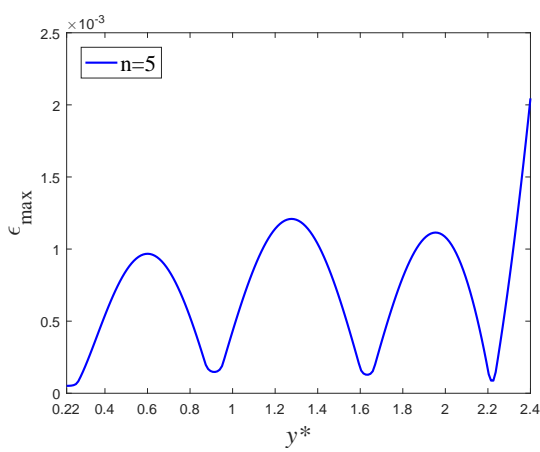

(e)

Figure 19: Distributions of the strains $\epsilon_{\max }(y)$ across the channel, $a^{*}=0.22$, $h^{*}=0.01$ in the hydroelastic waves with $k^{*}=0.5$.

The present study can be easily extended to cases involving free or simply supported edges at the lateral ends of the channel, through modifying the boundary conditions at $\tilde{y}=0$ in (A 2) to free or simply supported boundary conditions, and solving the corresponding eigen functions $\tilde{\psi}_{n}(\tilde{y})$. The present study is also planned to be extended further including asymmetric waves for different positions of a lead in the ice channel, and to be applied to the problem of a load moving along an ice channel either in the lead or on the ice cover near the lead. Motions of loads at critical speeds and generated stresses in the ice cover will receive special attention. We are unaware of results for the 
conditions, where two or more critical speeds merge. It would be challenging to describe the ice response to a load moving at such a special speed.

Acknowledgement. This research was started when the first author was visiting the School of Mathematics, University of East Anglia as an academic visitor in the period from December 2019 to May 2020. The authors are thankful to Prof. E.I. Parau for fruitfull discussions of nonlinear hydroelastic waves and to anonymous referees for their valuable suggestions on the interpretation of the obtained results and their presentations.

Funding. This work is supported by National Key R\&D Program of China (No. 2017YFE0111400) and by National Natural Science Foundation of China (Nos. 51979051, 51979056 and 51639004).

Declaration of Interests. The authors report no conflict of interest.

\section{Appendix A. Elastic modes $\psi_{n}(y)$}

Here we take the second equation of (3.2) as an example to illustrate how to solve the eigen-value problem in dimensionless variables. It is convenient to write the second equation of (3.2) in dimensionless variables, $\tilde{y}=(y-L) / \ell, \tilde{\psi}_{n}(\tilde{y})=\sqrt{\ell} \psi_{n}(\tilde{y} \ell+L)$, denoted by tilde. Here $\ell=L-a$ is the width of each ice plate. The dimensionless problem reads

$$
\begin{gathered}
\left(\frac{\mathrm{d}^{2}}{\mathrm{~d} \tilde{y}^{2}}-\tilde{k}^{2}\right)^{2} \tilde{\psi}_{n}=\tilde{\lambda}_{n}^{4} \tilde{\psi}_{n} \quad(-1<\tilde{y}<0), \\
\tilde{\psi}_{n}(0)=\tilde{\psi}_{n}^{\prime}(0)=0, \quad \tilde{\psi}_{n}^{\prime \prime}(-1)=\nu \tilde{k}^{2} \psi_{n}(-1), \quad \tilde{\psi}_{n}^{\prime \prime \prime}(-1)=(2-\nu) \tilde{k}^{2} \tilde{\psi}_{n}^{\prime}(-1),
\end{gathered}
$$

where $\tilde{k}=k \ell$ and $\tilde{\lambda}_{n}=\lambda_{n} \ell$. For $\tilde{k}=0$, this problem provides the shape functions of eigen vibrations of a cantilever beam, which is clamped at $\tilde{y}=0$ and free-free at $\tilde{y}=-1$, see Timoshenko \& Young (1955) The non-zero solutions of the problem (A 1), (A 2) for $\tilde{k}=0$ exist for $\lambda_{n}$, which satisfy the equation $\cos \tilde{\lambda}_{n} \cdot \cosh \tilde{\lambda}_{n}=-1$.

For $\tilde{k}>0$, the non-zero solutions of (A 1), (A 2) are given by (Eastham (1970))

$$
\tilde{\psi}_{n}(\tilde{y})=A_{n}\left[\frac{\sin q \tilde{y}}{q}-\frac{\sinh p \tilde{y}}{p}+Q\left(\tilde{\lambda}_{n}\right) \cos q \tilde{y}-Q\left(\tilde{\lambda}_{n}\right) \cosh p \tilde{y}\right],
$$

where $p=\sqrt{\tilde{\lambda}_{n}^{2}+\tilde{k}^{2}}, q=\sqrt{\tilde{\lambda}_{n}^{2}-\tilde{k}^{2}}$,

$$
Q\left(\tilde{\lambda}_{n}\right)=\frac{\left[\tilde{\lambda}_{n}^{2}+(1-\nu) \tilde{k}^{2}\right] \frac{\sinh p}{p}+\left[\tilde{\lambda}_{n}^{2}-(1-\nu) \tilde{k}^{2}\right] \frac{\sin q}{q}}{\left[\tilde{\lambda}_{n}^{2}+(1-\nu) \tilde{k}^{2}\right] \cosh p+\left[\tilde{\lambda}_{n}^{2}-(1-\nu) \tilde{k}^{2}\right] \cos q},
$$

and the coefficients $A_{n}$ are determined by the second equation of (3.3),

$$
\begin{aligned}
\frac{1}{A_{n}^{2}} & =-2 Q\left[\frac{\sin ^{2} q}{2 q^{2}}+\frac{\sinh ^{2} p}{2 p^{2}}-\frac{\sinh p \sin q}{p q}\right] \\
& +Q^{2}\left[1+\frac{\sin q \cos q}{2 q}+\frac{\sinh p \cosh p}{2 p}-2 \frac{q \cosh p \sin q+p \sinh p \cos q}{p^{2}+q^{2}}\right] \\
& +\left[\frac{p^{2}-q^{2}}{2 p^{2} q^{2}}+\frac{\sinh p \cosh p}{2 p^{3}}-\frac{\sin q \cos q}{2 q^{3}}-2 \frac{p \cosh p \sin q-q \sinh p \cos q}{p q\left(p^{2}+q^{2}\right)}\right] .
\end{aligned}
$$

These non-zero solutions exist for $\tilde{\lambda}_{n}$ which are real and positive roots of the equation 


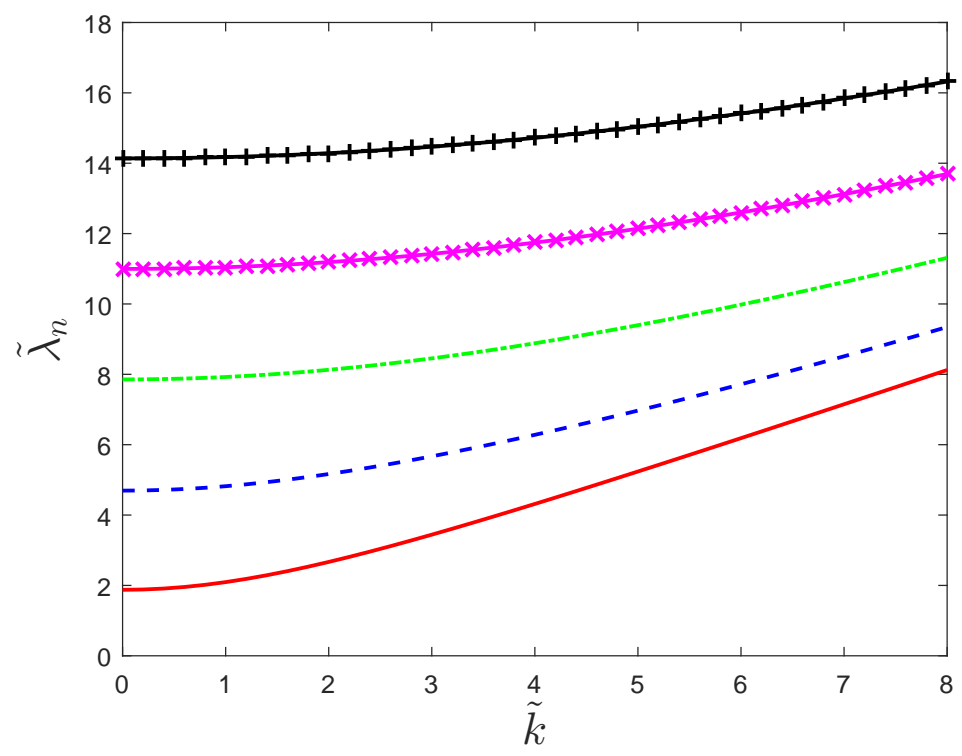

Figure 20: The dimensionless spectral parameters $\tilde{\lambda}_{n}$ as functions of the dimensionless wave number $\tilde{k}$ for $\nu=0.3$ and $n=1,2,3,4,5$.

$$
\frac{\tilde{\lambda}_{n}^{4}-(1-\nu)^{2} \tilde{k}^{4}}{\tilde{\lambda}_{n}^{4}+(1-\nu)^{2} \tilde{k}^{4}}+\cosh p \cos q=\left[\tilde{k}^{2}-\frac{2 \tilde{\lambda}_{n}^{4}(1-\nu) \tilde{k}^{2}}{\tilde{\lambda}_{n}^{4}+(1-\nu)^{2} \tilde{k}^{4}}\right] \frac{\sinh p}{p} \frac{\sin q}{q} .
$$

Equations (A 3)-(A 6) are writen for $\tilde{\lambda}_{n}>\tilde{k}$. For $\tilde{\lambda}_{n}=\tilde{k}$, one needs to change $\cos \left(\sqrt{\tilde{\lambda}_{n}^{2}-\tilde{k}^{2}}\right)$ and $\sin \left(\sqrt{\tilde{\lambda}_{n}^{2}-\tilde{k}^{2}}\right) /\left(\sqrt{\tilde{\lambda}_{n}^{2}-\tilde{k}^{2}}\right)$ to 1 . For $\tilde{\lambda}_{n}<\tilde{k}$, one needs to change $\cos \left(\sqrt{\tilde{\lambda}_{n}^{2}-\tilde{k}^{2}}\right)$ to $\cosh \left(\sqrt{\tilde{k}^{2}-\tilde{\lambda}_{n}^{2}}\right)$ and $\sin \left(\sqrt{\tilde{\lambda}_{n}^{2}-\tilde{k}^{2}}\right) /\left(\sqrt{\tilde{\lambda}_{n}^{2}-\tilde{k}^{2}}\right)$ to $\sinh \left(\sqrt{\tilde{k}^{2}-\tilde{\lambda}_{n}^{2}}\right) /\left(\sqrt{\tilde{k}^{2}-\tilde{\lambda}_{n}^{2}}\right)$. The roots of (A 6$), \tilde{\lambda}_{n}(\tilde{k}), n=1,2,3,4,5$, are shown in figure 20 as functions of the dimensionless wave number $\tilde{k}$ for $\nu=0.3$. Note that $\tilde{\lambda}_{n+1}(\tilde{k})>\tilde{\lambda}_{n}(\tilde{k})$ for $\tilde{k} \geqslant 0$ and $n \geqslant 1$. In particular, $\tilde{\lambda}_{1}(\tilde{k})=\tilde{k} \approx 23.413$ for $\nu=0.3$.

The shape functions $\tilde{\psi}_{n}(\tilde{y})$ defined by (A 3)-(A 6$)$ are shown in figure 21 for $\tilde{k}=1$, $\nu=0.3$ and $n=1,2,3,4,5$. These shape functions, $\tilde{\psi}_{n}(\tilde{y})$, have $n-1$ zeros inside the interval $(-1,0)$, where $\tilde{y}=0$ corresponds to the clamped edge of the ice plate.

\section{Appendix B. The integrals $\tilde{A}_{n r}$ from section 3}

The method of separating variables applied to the boundary problem (3.7) and (3.8) provides

$$
\Phi_{n}(y, z)=\sum_{m=1}^{\infty} D_{n m} \frac{\cosh \left[\sqrt{k^{2}+\mu_{m}^{2}}(z+H)\right]}{\cosh \left[\sqrt{k^{2}+\mu_{m}^{2}} H\right]} \cos \left(\mu_{m} y\right),
$$

where $\mu_{m}=\pi(m-1) / L$, the coefficients $D_{n m}$ are determined using the boundary condition (3.13), 


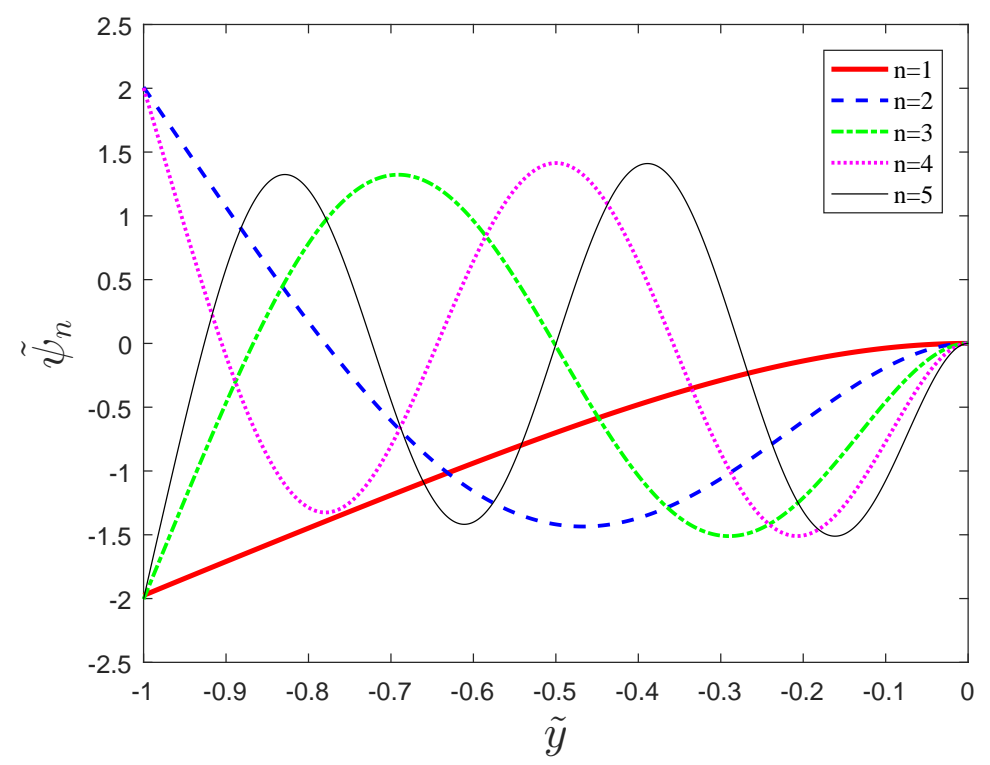

Figure 21: The dimensionless normalised shape functions $\tilde{\psi}_{n}(\tilde{y})$ for $\tilde{k}=1$, $\nu=0.3$, and $n=1,2,3,4,5$.

$$
\sum_{m=1}^{\infty} D_{n m}\left(\sqrt{k^{2}+\mu_{m}^{2}} \tanh \left[\sqrt{k^{2}+\mu_{m}^{2}} H\right]-\frac{\omega^{2}}{g}\right) \cos \left(\mu_{m} y\right)=\psi_{n}(|y|) \mathcal{H}\left(y^{2}-a^{2}\right),
$$

$\mathcal{H}(x)$ is the Heaviside function, which is equal to zero for negative $x$ and one for positive $x$. The functions $\cos \left(\mu_{m} y\right), m \geqslant 1$, are orthogonal in the interval $-L<y<L$,

$$
\int_{-L}^{L} \cos \left(\mu_{m} y\right) \cos \left(\mu_{r} y\right) \mathrm{d} y= \begin{cases}0, & r \neq m \\ L, & r=m \neq 1 \\ 2 L, & r=m=1\end{cases}
$$

Multiplying both sides of (B 2) by $\cos \left(\mu_{r} y\right)$ and integrating the result in $y$ from $-L$ to $L$ using the relations (B 3), we find

$$
D_{n m}=\frac{\epsilon_{m}}{L\left[\sqrt{k^{2}+\mu_{m}^{2}} \tanh \left[\sqrt{k^{2}+\mu_{m}^{2}} H\right]-\omega^{2} / g\right]} \int_{a}^{L} \psi_{n}(y) \cos \left(\mu_{m} y\right) \mathrm{d} y,
$$

where $\epsilon_{1}=1$ and $\epsilon_{m}=2$ for $m \geqslant 2$. The denominators in (B 4) are not equal to zero in our computations for a channel with ice cover and a lead because we do not consider the frequencies $\omega=\omega_{m}^{(f)}(k)=\sqrt{g \sqrt{k^{2}+\mu_{m}^{2}} \tanh \left[\sqrt{k^{2}+\mu_{m}^{2}} H\right]}$, which correspond to the frequencies of water waves in the channel without ice.

Denote the integrals in (B 4) by $S_{n m}$. Then equations (B 1) and (B 4) give the integrals from section 3 , 


$$
\tilde{A}_{n r}=\int_{a}^{L} \Phi_{n}(y, 0) \psi_{r}(y) \mathrm{d} y=\sum_{m=1}^{\infty} \frac{\epsilon_{m} S_{n m} S_{r m}}{L\left[\sqrt{k^{2}+\mu_{m}^{2}} \tanh \left[\sqrt{k^{2}+\mu_{m}^{2}} H\right]-\omega^{2} / g\right]} .
$$

With the help of integration by parts one can show that $S_{n m} \sim-\psi_{n}(a) \sin \left(\mu_{m} a\right) / \mu_{m}$ as $m \rightarrow \infty$. Therefore, the terms in the series (B 5) decay as $m^{-3}$ for $m \rightarrow \infty$. The integrals $S_{n m}$ are evaluated by multiplying both sides of the second equation of $(3.2)$ by $\cos \left(\mu_{m} y\right)$ and integrating the result in $y$ from $a$ to $L$ by parts using the edge conditions from the second equation of (3.2), which gives

$$
\int_{a}^{L}\left(\frac{\mathrm{d}^{2}}{\mathrm{~d} y^{2}}-k^{2}\right)^{2} \psi_{n} \cos \left(\mu_{m} y\right) \mathrm{d} y=\int_{a}^{L} \lambda_{n}^{4} \psi_{n} \cos \left(\mu_{m} y\right) \mathrm{d} y
$$

and

$$
S_{n m}=\frac{(-1)^{m} \psi_{n}^{\prime \prime \prime}(L)+\left(\mu_{m}^{2}+\nu k^{2}\right) \cos \left(\mu_{m} a\right) \psi_{n}^{\prime}(a)+\mu_{m}\left[(2-\nu) k^{2}+\mu_{m}^{2}\right] \sin \left(\mu_{m} a\right) \psi_{n}(a)}{\lambda_{n}^{4}-\left(k^{2}+\mu_{m}^{2}\right)^{2}} .
$$

Using the dimensionless variable from Appendix $\mathrm{A}, y=\tilde{y} \ell+L$, where $\ell=L-a$, mutiplying (B 7) by $\ell^{4}$ in the numerator and denominator, and denoting $\mu_{m} \ell=\pi(m-$ 1) $(1-a / L)$ by $\tilde{\mu}_{m}$, we find

$$
\begin{aligned}
S_{n m}=\frac{\sqrt{\ell}}{\tilde{\lambda}_{n}^{4}-\left(\tilde{k}^{2}+\tilde{\mu}_{m}^{2}\right)^{2}}\left[(-1)^{m} \tilde{\psi}_{n}^{\prime \prime \prime}(0)\right. & +\tilde{\mu}_{m}\left[(2-\nu) \tilde{k}^{2}+\tilde{\mu}_{m}^{2}\right] \sin \left(\mu_{m} a\right) \tilde{\psi}_{n}(-1) \\
& \left.+\left(\tilde{\mu}_{m}^{2}+\nu \tilde{k}^{2}\right) \cos \left(\mu_{m} a\right) \tilde{\psi}_{n}^{\prime}(-1)\right]
\end{aligned}
$$

where the modes $\tilde{\psi}_{n}(y)$ and the eigenvalues $\tilde{\lambda}_{n}$ are defined in Appendix A.

\section{REFERENCES}

Batyaev, E. A. \& Khabakhpasheva, T. I. 2015 Hydroelastic waves in a channel covered with a free ice sheet. Fluid Dynamics. 50 (6), 775-788.

Beltaos, S. 2004 Wave-generated fractures in river ice covers. Cold Regions Science and Technology. 40 (3), 179-191.

Brocklehurst, P., Korobkin, A. \& Parau, E. I. 2011 Hydroelastic wave diffraction by a vertical cylinder. Philosophical Transactions of the Royal Society A: Mathematical, physical and engineering sciences 369 (1947), 2832-2851.

Chung, H. \& Linton, C. M. 2005 Reflection and transmission of waves across a gap between two semi-infinite elastic plates on water. Q. J. Mech. Appl. Maths. 58 (1), 1-15.

DALY, S. F. 1993 Wave propagation in ice-covered channels. Journal of Hydraulic Engineering 119 (8), 895-910.

DALY, S. F. 1995 Fracture of river ice covers by river waves. Journal of Cold Regions Engineering. 9 (1), 41-52.

Dinvay, E., Kalisch, H. \& Parau, E. I. 2019 Fully dispersive models for moving loads on ice sheets. Journal of Fluid Mechanics 876, 122-149.

Eastham, M. S. P. 1970 Theory of Ordinary Differential Equations. London, Van Nostrand Reinhold.

Fuamba, M., Bouannani, N. \& Marche, C. 2007 Modeling of dam break wave propagation in a partially ice-covered channel. Advances in Water Resources. 30 (12), 2499-2510.

Guyenne, P. \& PARAu, E. I. 2012 Computations of fully nonlinear hydroelastic solitary waves on deep water. J. Fluid Mech. 713, 307-329. 
Guyenne, P. \& PARAu, E. I. 2017 Numerical study of solitary wave attenuation in a fragmented ice sheet. Physical Review Fluids 2 (3).

Korobkin, A. A., Khabakhpasheva, T. I. \& Papin, A. A. 2014 Waves propagating along a channel with ice cover. European Journal of Mechanics-B/Fluids. 47, 166-175.

Marchenko, A. 1997 Resonance interactions of waves in an ice channel. Journal of Applied Mathematics and Mechanics. 61 (6), 931-940.

Marchenko, A. 1999 Parametric excitation of flexural-gravity edge waves in the fluid beneath an elastic ice sheet with a crack. European Journal of Mechanics - B/Fluids 18 (3), 511525.

Nzokou, F., Morse, B. \& Quach-Thanh, T. 2009 River ice cover flexure by an incoming wave. Cold Regions Science and Technology 55 (2), 230-237.

Nzokou, F., Morse, B., Robert, J.-L., Richard, M. \& Tossou, E. 2011 Water wave transients in an ice-covered channel. Canadian Journal of Civil Engineering. 38 (4), 404414.

PARAu, E. I. \& DiAs, F. 2002 Nonlinear effects in the response of a floating ice plate to a moving load. J. Fluid Mech. 460, 281-305.

Porter, R. 2018 Trapping of waves by thin floating ice floes. Q. J. Mech. Appl. Maths. 71 (4), 463-483.

REN, K., Wu, G. X. \& LI, Z. F. 2020 Hydroelastic waves propagating in an ice-covered channel. J. Fluid Mech. 886 (A18), 1-24.

ShI, Y. Y., LI, Z. F. \& Wu, G. X. 2019 Interaction of wave with multiple wide polynyas. Physics of Fluids 31 (6), 067111.

Shishmarev, K. A., Khabakhpasheva, T. I. \& Korobkin, A. A. 2016 The response of ice cover to a load moving along a frozen channel. Applied Ocean Research 59, 313-326.

Squire, V. A. 2007 Of ocean waves and sea-ice revisited. Cold Regions Science and Technology 49 (2), 110-133.

Squire, V. A. 2011 Past, present and impendent hydroelastic challenges in the polar and subpolar seas. Philosophical Transactions of the Royal Society A: Mathematical, Physical and Engineering Sciences 369 (1947), 2813-2831.

Timoshenko, S. \& Young, D. H. 1955 Vibration problems in engineering, 3rd edition. D. van Nostrand company.

Wang, Z., Parau, E. I., Milewski, P. A. \& Vanden-Broeck, J.-M. 2014 Numerical study of interfacial solitary waves propagating under an elastic sheet. Proc Math Phys Eng 470 (2168), 20140111.

Williams, T. D. \& Squire, V. A. 2006 Scattering of flexural-gravity waves at the boundaries between three floating sheets with applications. J. Fluid Mech. 569, 113-140.

XIA, X. \& Shen, H. T. 2002 Nonlinear interaction of ice cover with shallow water waves in channels. J. Fluid Mech. 467, 259-268. 